\title{
On the ground-state energy of the finite sine-Gordon ring
}

\author{
Sergei B. Rutkevich \\ Fakultät für Mathematik und Naturwissenschaften, Bergische Universität Wuppertal, \\ 42097 Wuppertal, Germany. \\ E-mail: rutkevich@uni-wuppertal.de
}

\begin{abstract}
The Casimir scaling function characterising the ground-state energy of the sine-Gordon model in a finite circle has been studied analytically and numerically both in the repulsive and attractive regimes. The numerical calculations of the scaling function at several values of the coupling constant were performed by the iterative solution of the Destri-de Vega nonlinear integral equations. The ultraviolet asymptotics of the Casimir scaling functions was calculated by perturbative solution of these equations, and by means of the perturbed conformal field-theory technique, and compared with numerical results.
\end{abstract}

\section{Introduction}

The ground state energy $E(L)$ of the $(1+1)$-dimensional massive relativistic quantum field theory (QFT) defined in the circle $\mathbb{R} / L$ can be represented as:

$$
E(L)=L \mathcal{E}_{b}+\frac{Y(u)}{L} .
$$

Here $\mathcal{E}_{b}$ is the bulk energy density, $L$ is the circle circumference, and $Y(u)$ is the Casimir finite-size scaling function depending on the scaling parameter $u=m L$, where $m$ is the mass of the lightest particle in the theory. This well-known fact (see e.g. [1] and references therein) follows immediately from simple dimensional arguments. It is well known also [2], that the free energy per unit length $f(T)$ at a non-zero temperature $T>0$ of the same QFT defined in the infinite line $\mathbb{R}$ can be also expressed in terms of the Casimir scaling function:

$$
f(T)=\mathcal{E}_{b}+T^{2} Y(u),
$$

with the different meaning of the scaling parameter $u=m / T$, however.

The ground- and excited-state finite-size spectra in $(1+1)$-dimensional quantum field theories attract much interest in recent decades. This interest stems from several reasons. First, studying of such spectra gives a deep insight into the structure of integrable QFT, yielding information about their monodromy properties [3], integrals of motions [4], and the renormalization group flow [5]. Second, investigation of the finitesize scaling is crucial for correct interpretation of the results of computer simulations, 
which are typically performed on finite-size systems. And third, the relativistic $(1+1)$ dimensional QFT can describe the dynamic and thermodynamic properties of the quazione-dimensional magnetic crystals in the scaling region near their continuous quantum phase transition points. Accordingly, the appropriate universal Casimir scaling function $Y(u)$ should determine due to (2) the free-energy temperature dependence of such a crystal in the scaling regime [6].

Different methods have been used to study the finite-size scaling functions, such as the conformal perturbation theory [7, 8, 9], and the truncated conformal space approach [10, 11. A rather effective approach to the finite-size scaling problem in integrable quantum spin chains and QFTs was introduced in the early 90-th by Batchelor, Klümper, and Pearce [12, 13, and later used by Destri and de Vega for the sine-Gordon model. In the pioneered works [14, 15], Destri and de Vega analysed the inhomogeneous lightcone version of the six-vertex model, and derived in the continuous limit the nonlinear integral equation describing the ground-state energy of the sine-Gordon finite circle. Its Euclidean dynamics evolves in the cylinder having one spatial, and one (Euclidean) time direction. An alternative viewpoint [15, 2] on such a quantum dynamics is possible, in which one treats the compact direction on the cylinder as the Matsubara direction. In this alternative picture, the nonlinear integral equations are interpreted as the Thermodynamic Bethe Ansatz (TBA) equations, which determine the free energy of the infinite system at a non-zero temperature. Note, that similar nonlinear integral TBA equations for different spin-chain models were obtained earlier by Klümper, Batchelor, and Pearce [13, 16. The approach based on the nonlinear integral equations was later generalised and applied to describe the finite-size excited-energy spectra in integrable QFT. The latter subject attracted much attention and has been studied by many authors [10, 11, 17, 18, 19, 20, 21, 22].

The important advantage of the Destri-de Vega (DDV) nonlinear integral equations for the sine-Gordon model is that they not only make possible rather accurate numerical calculations of the finite-size energy spectra [17], but also can be used to derive perturbatively the asymptotic expansions for these spectra in different limiting cases. For the ground-state energy $E(L)$ of the sine-Gordon circle of length $L$, two such expansions at $L \rightarrow \infty$, and at $L \rightarrow 0$ were first studied by Destri and de Vega in [15. Their perturbative analysis in the infrared (recursive) limit $L \rightarrow \infty$ was rather straightforward and will not be discussed here. The asymptotical analysis of the DDV equations in the opposite ultraviolet (conformal) limit $L \rightarrow 0$, which is much more difficult, was presented in Section 7 of [15]. The final result (1.13) of this Section for the asymptotic expansion of the ground state energy $E(L)$ in this limit can be written as:

$$
E(L) \underset{L \rightarrow 0}{=} L \mathcal{E}_{b}-\frac{\pi}{6 L}-\frac{m^{2} L}{4} \cot \frac{\pi^{2}}{2 \gamma}+\frac{1}{L} \sum_{n=0}^{\infty} C_{n}(\gamma)(m L)^{4 n \gamma / \pi}
$$

Here $m$ is the soliton mass, $\gamma \in(0, \pi)$ is the real parameter simply related with the coupling constant $\beta$, see equation (6) below. The "repulsive" and "attractive" regimes of the sine-Gordon model are realized at $0<\gamma<\pi / 2$, and at $\pi / 2<\gamma<\pi$, respectively. 
The bulk energy density $\mathcal{E}_{b}$ in the first term in the right-hand side contains the nonuniversal ultraviolet-divergent contribution. The second term describes the leading finite-size correction, which agrees with the Conformal Field Theory (CFT) prediction for the case of the central charge $c=1$. The third and fourth terms in the right-hand side of (3) represent the sub-leading finite-size corrections.

It turns out, however, that the original derivation of the expansion (3) described in Section 7 of [15] was not completely consistent being essentially based on a mistaken assumption. In the present work we reconsider derivation of expansion (3) for the ground state energy in the ultraviolet limit $L \rightarrow 0$ in order to fill this gap. Combing asymptotical analysis of the nonlinear TBA equations with the perturbative CFT calculation, we confirm, that expansion (3) holds both in the attractive and repulsive regimes at all generic $\gamma \in(0, \pi)$, apart from the points $\gamma_{l}=\frac{\pi}{2 l}, l=1,2, \ldots$ At these exceptional points, the diverging factor $\cot \frac{\pi^{2}}{2 \gamma}$ in the right-hand side of $(3)$ should be replaced by the factor $\frac{2}{\pi l} \ln u$. It is shown, that the sum in the right-hand side of (3) starts in fact from the $n=1$ term, and for the coefficient $C_{1}(\gamma)$ we obtained the following explicit expression:

$$
C_{1}(\gamma)=-\frac{(16 \pi)^{1-2 \gamma / \pi}}{4}\left[\frac{\Gamma\left(\frac{\pi}{2 \gamma}\right)}{\Gamma\left(\frac{\pi}{2 \gamma}-\frac{1}{2}\right)}\right]^{4 \gamma / \pi}\left[\frac{\Gamma\left(1-\frac{\gamma}{\pi}\right)}{\Gamma\left(\frac{\gamma}{\pi}\right)}\right]^{4} \frac{\Gamma\left(\frac{2 \gamma}{\pi}-1\right)}{\Gamma\left(2-\frac{2 \gamma}{\pi}\right)} .
$$

This formula represents the main result of the present work. Note, that the finite-size correction term $L^{-1} C_{1}(\gamma)(m L)^{4 \gamma / \pi}$ dominates over the term $-\frac{m^{2} L}{4} \cot \frac{\pi^{2}}{2 \gamma}$ in the repulsive regime $0<\gamma<\pi / 2$ at $m L \rightarrow 0$, while the cotangent term excesses the infinite sum in the right-hand side of (3) in the attractive case $\pi / 2<\gamma<\pi$.

The rest of the paper is organised as follows. In the next Section, we recall few necessary facts about the quantum sine-Gordon model. Section 3 is addressed to the sine-Gordon model in the cylindric geometry. We describe there two versions of the nonlinear integral equations derived by Destri and de Vega [15], then critically review their asymptotical analysis of the second (TBA) version of these equations in the ultraviolet limit. It is shown, that this analysis in [15] was inconsistent and requires revision. The improved perturbative calculations of the Casimir scaling function $Y(u \mid \gamma)$ in the ultraviolet limit $u \rightarrow 0$ are presented in Section 4. In Section 5, we describe the results of numerical calculations of the Casimir scaling function $Y(u \mid \gamma)$ obtained by the iterative solution of the DDV integral equations at different values of the parameter $\gamma$. At small $u \ll 1$, these numerical results display a nice agreement with the analytical asymptotic dependencies obtained in Section 4. Finally, there are three Appendices. In Appendices A and B we describe two alternative analytical calculations of the Casimir scaling function $Y(u \mid \gamma)$ in the ultraviolet limit $u \ll 1$. In Appendix A we exploit to this end the small- $u$ asymptotical analysis of the nonlinear TBA equations, while in Appendix B we use the perturbative CFT technique. In Appendix C we recall some results of [4] relating to the "massless" case $u=0$ of the DDV equation, and show that our findings are consistent with these results. 


\section{Quantum sine-Gordon model}

The two-dimensional sine-Gordon model can be defined by the action [23],

$$
\mathcal{A}_{S G}=\frac{1}{16 \pi} \int\left(\partial_{a} \varphi\right)^{2} d^{2} x-2 \mu \int \cos (\beta \varphi) d^{2} x
$$

where $\varphi(x)$ is the scalar field in the two-dimensional Euclidean space-time with the coordinates $x^{a}=\left(x^{0}, x^{1}\right)$, and $0<\beta<1$ is the real parameter. We shall use also three related parameters $0<\gamma_{s}<\infty, 0<\gamma<\pi$, and $0<\xi<\infty$,

$$
\gamma_{s}=\frac{8 \pi \beta^{2}}{1-\beta^{2}}, \quad \gamma=\pi\left(1-\beta^{2}\right), \quad \xi=\frac{\beta^{2}}{1-\beta^{2}} .
$$

The sine-Gordon model can be viewed as the perturbation of the Gaussian field theory by the exponential operators $\exp ( \pm i \beta \varphi)$ having the scaling dimension

$$
\mathrm{x}=2 \beta^{2}=\frac{2 \xi}{\xi+1}=\frac{2(\pi-\gamma)}{\pi} .
$$

These fields will be normalised according to the conventional in the CFT condition [23],

$$
\left\langle e^{i \beta \varphi(x)} e^{-i \beta \varphi(0)}\right\rangle_{\mu=0}=\frac{1}{|x|^{2 \mathrm{x}}} .
$$

The particle content and the scattering properties of the sine-Gordon model are well known [24]. In the repulsive regime at $\frac{1}{2}<\beta^{2}<1$, the model contains only the soliton and antisoliton excitations, which are the massive relativistic particles. Accordingly, their energy and momentum read,

$$
\epsilon(\alpha)=m \cosh \alpha, \quad p(\alpha)=m \sinh \alpha,
$$

where $\alpha$ is the particle rapidity. In the attractive regime at $0<\beta^{2}<\frac{1}{2}$, the bound states of solitons and antisolitons also emerge in the theory. The dimensional coupling constant $\mu$ is related to the soliton mass $m$ as follows:

$$
\mu=\kappa(\xi) m^{2 /(\xi+1)} \text {. }
$$

The constant $\kappa(\xi)$ was found by Al. B. Zamolodchikov [23]:

$$
\kappa(\xi)=\frac{1}{\pi} \frac{\Gamma\left(\frac{\xi}{\xi+1}\right)}{\Gamma\left(\frac{1}{\xi+1}\right)}\left[\frac{\sqrt{\pi} \Gamma\left(\frac{\xi+1}{2}\right)}{2 \Gamma\left(\frac{\xi}{2}\right)}\right]^{2 /(\xi+1)} .
$$

The scattering matrix in the sine-Gordon model is known due to A. B. Zamolodchikov [24]. In particular, the integral form of the soliton-soliton scattering amplitude reads [25],

$$
S\left(\alpha, \gamma_{s}\right)=-\exp \left[-i \int_{0}^{\infty} \frac{d y}{y} \frac{\sin (2 \alpha y) \sinh \left[\left(\pi-\frac{\gamma_{s}}{8}\right) y\right]}{\cosh (\pi y) \sinh \left(\gamma_{s} y / 8\right)}\right] .
$$

This scattering amplitude satisfies the following equality,

$$
S\left(\alpha, \gamma_{s}\right) S\left(\alpha+i \pi, \gamma_{s}\right)=-\frac{\sinh \left(8 \pi \alpha / \gamma_{s}\right)}{\sinh \left[8 \pi(\alpha+i \pi) / \gamma_{s}\right]} .
$$


On the ground-state energy of the finite sine-Gordon ring

Note, that the XYZ spin-1/2 chain model defined by the Hamiltonian

$$
H=\frac{1}{2} \sum_{j}\left(J_{x} \sigma_{j}^{x} \sigma_{j+1}^{x}+J_{y} \sigma_{j}^{y} \sigma_{j+1}^{y}+J_{z} \sigma_{j}^{z} \sigma_{j+1}^{z}\right),
$$

is known to become equivalent to the sine-Gordon model in the continuous limit

$$
0<\left|J_{z}\right|<J_{x}<J_{y}, \quad J_{y}-J_{x} \ll J_{x}, \quad J_{z}=J_{x} \cos \gamma .
$$

\section{Sine-Gordon model on the cylinder and the DDV equation}

Let us now turn to the sine-Gordon model defined on the torus $\mathcal{T}$,

$$
\mathcal{T}=\left\{x \in \mathcal{T} \mid 0<x^{0}<L^{\prime}, 0<x^{1}<L\right\}
$$

with the periodical boundary conditions,

$$
\varphi\left(x^{0}, x^{1}\right)=\varphi\left(x^{0}, x^{1}+L\right)=\varphi\left(x^{0}+L^{\prime}, x^{1}\right),
$$

and proceed to the limit $L^{\prime} \rightarrow \infty$ corresponding to the cylindric geometry. The partition function

$$
Z\left(\mu, L, L^{\prime}\right)=\int D[\varphi] \exp \left(-\mathcal{A}_{G}[\varphi]\right) \exp \left\{2 \mu \int_{\mathcal{T}} d^{2} x \cos [\beta \varphi(x)]\right\}
$$

can be written in this limit as

$$
Z\left(\mu, L, L^{\prime}\right)=\exp \left[-L^{\prime} E(\mu, L)+O(1)\right]
$$

where $\mathcal{A}_{G}[\varphi]$ is the action of the Gaussian free-field theory, and $E(\mu, L)$ is the groundstate energy of the sine-Gordon model in the circle of length $L$. The latter can be represented as

$$
E(\mu, L)=L \mathcal{E}_{b}+\frac{Y(u \mid \gamma)}{L},
$$

where $\mathcal{E}_{b}=\mathcal{E}_{b}(m, \gamma)$ is the bulk energy density, and $Y(u \mid \gamma)$ is the Casimir scaling function, which depends on the scaling parameter $u=m L$.

The scaling function $Y(u \mid \gamma)$ has the following explicit representation

$$
Y(u \mid \gamma)=-\frac{u}{\pi} \int_{-\infty}^{\infty} d \alpha \sinh \alpha \cdot \operatorname{Im} \ln [1+\mathfrak{f}(\alpha+i 0 \mid u, \gamma)]
$$

where $\mathfrak{f}(\alpha \mid u, \gamma)$ is the solution of the DDV nonlinear integral equation

$$
\begin{aligned}
& -i \ln \mathfrak{f}(\alpha \mid u, \gamma)=u \sinh \alpha+ \\
& 2 \int_{-\infty}^{\infty} d \alpha^{\prime} G\left(\alpha-\alpha^{\prime} \mid \gamma\right) \operatorname{Im} \ln \left[1+\mathfrak{f}\left(\alpha^{\prime}+i 0 \mid u, \gamma\right)\right]
\end{aligned}
$$

The integral kernel $G(\alpha \mid \gamma)$ in the right-hand side of $(21)$ is related with the solitonsoliton scattering amplitude (12),

$$
G(\alpha \mid \gamma)=\left.\frac{1}{2 \pi i} \frac{\partial \ln S\left(\alpha, \gamma_{s}\right)}{\partial \alpha}\right|_{\gamma_{s}=8 \pi\left(\frac{\pi}{\gamma}-1\right)}=\frac{1}{4 \pi} \int_{-\infty}^{\infty} d k \frac{\cos (\alpha k) \sinh \left[\left(\frac{\pi^{2}}{2 \gamma}-\pi\right) k\right]}{\cosh \left(\frac{\pi k}{2}\right) \sinh \left[\left(\frac{\pi^{2}}{2 \gamma}-\frac{\pi}{2}\right) k\right]}
$$


Note the equality following from (13),

$$
G(\alpha+i \pi \mid \gamma)=-G(\alpha \mid \gamma)-g(\alpha \mid \gamma)
$$

where

$$
g(\alpha \mid \gamma)=\frac{i \gamma}{2 \pi(\pi-\gamma)}\left[\operatorname{coth}\left(\frac{\gamma \alpha}{\pi-\gamma}\right)-\operatorname{coth}\left(\frac{\gamma(i \pi+\alpha)}{\pi-\gamma}\right)\right]
$$

The exact representation (20)-(22) for the Casimir scaling function of the sine-Gordon model was obtained by Destri and de Vega [14, 15] by analysis of the Bethe-Ansatz equations for the six-vertex model in the light-cone approach. Later Fioravanti and Rossi [26] derived the same representation for $Y(u \mid \gamma)$ from the Bethe-Ansatz solution of the XYZ spin chain in the scaling limit (15).

The integral representation (20)-22 for the Casimir scaling function $Y(u \mid \gamma)$ applies to the sine-Gordon model in the complete range $0<\gamma<\pi$ of the parameter $\gamma$. In the repulsive regime $0<\gamma<\pi / 2$, it can be modified [15] by means of the analytical continuation to the form

$$
Y(u \mid \gamma)=-\frac{u}{\pi} \int_{-\infty}^{\infty} d \alpha \cosh \alpha \operatorname{Re} \ln \left[1+e^{-\varepsilon(\alpha \mid u, \gamma)}\right],
$$

where the function $\varepsilon(\alpha \mid u, \gamma)=-\ln \mathfrak{f}(\alpha+i \pi / 2 \mid u, \gamma)$ (the pseudoenergy) solves the system of the TBA integral equations,

$$
\begin{aligned}
& \varepsilon(\alpha \mid u, \gamma)=u \cosh \alpha-\int_{-\infty}^{\infty} d \alpha^{\prime} G\left(\alpha-\alpha^{\prime} \mid \gamma\right) \ln \left[1+e^{-\varepsilon\left(\alpha^{\prime} \mid u, \gamma\right)}\right] \\
& +\int_{-\infty}^{\infty} d \alpha^{\prime} G\left(\alpha-\alpha^{\prime}+i \pi-i 0 \mid \gamma\right) \ln \left[1+e^{-\bar{\varepsilon}\left(\alpha^{\prime} \mid u, \gamma\right)}\right] \\
& \bar{\varepsilon}(\alpha \mid u, \gamma)=u \cosh \alpha-\int_{-\infty}^{\infty} d \alpha^{\prime} G\left(\alpha-\alpha^{\prime} \mid \gamma\right) \ln \left[1+e^{-\bar{\varepsilon}\left(\alpha^{\prime} \mid u, \gamma\right)}\right] \\
& +\int_{-\infty}^{\infty} d \alpha^{\prime} G\left(\alpha-\alpha^{\prime}-i \pi+i 0 \mid \gamma\right) \ln \left[1+e^{-\varepsilon\left(\alpha^{\prime} \mid u, \gamma\right)}\right]
\end{aligned}
$$

and $\bar{\varepsilon}(\alpha \mid u, \gamma)$ is the complex conjugate of $\varepsilon(\alpha \mid u, \gamma)$. Exploiting equality (23), these equations can be rewritten in the equivalent form,

$$
\begin{aligned}
& \varepsilon(\alpha \mid u, \gamma)=u \cosh \alpha-2 \int_{-\infty}^{\infty} d \alpha^{\prime} G\left(\alpha-\alpha^{\prime} \mid \gamma\right) \operatorname{Re} \ln \left[1+e^{-\varepsilon\left(\alpha^{\prime} \mid u, \gamma\right)}\right] \\
& -\int_{-\infty}^{\infty} d \alpha^{\prime} g\left(\alpha-\alpha^{\prime}-i 0 \mid \gamma\right) \ln \left[1+e^{-\bar{\varepsilon}\left(\alpha^{\prime} \mid u, \gamma\right)}\right] \\
& \bar{\varepsilon}(\alpha \mid u, \gamma)=u \cosh \alpha-2 \int_{-\infty}^{\infty} d \alpha^{\prime} G\left(\alpha-\alpha^{\prime} \mid \gamma\right) \operatorname{Re} \ln \left[1+e^{-\bar{\varepsilon}\left(\alpha^{\prime} \mid u, \gamma\right)}\right] \\
& -\int_{-\infty}^{\infty} d \alpha^{\prime} g\left(\alpha-\alpha^{\prime}+i 0 \mid \gamma\right) \ln \left[1+e^{-\varepsilon\left(\alpha^{\prime} \mid u, \gamma\right)}\right]
\end{aligned}
$$

In Section 7.3 of their article [15], Destri and de Vega performed the asymptotical analysis of the nonlinear integral equations (26) in order to describe the behaviour of the scaling function $Y(u \mid \gamma)$ at large and small $u$. Since equations (26) can be used only in the repulsive regime $0<\gamma<\pi / 2$, their analysis could be related to this regime only. 
In what follows, we shall recall the main steps of this analysis in the ultraviolet limit $u \rightarrow 0$.

Destri and de Vega wrote the solution of equations (26) at $u \ll 1$ in the form,

$$
\varepsilon(\alpha \mid u, \gamma)=\varepsilon_{k}(\alpha-R(u) \mid \gamma)+\varepsilon_{k}(-\alpha-R(u) \mid \gamma)+\eta(\alpha \mid u, \gamma),
$$

where $R(u)=\ln (2 / u)$, and the function

$$
\varepsilon_{k}(\alpha \mid \gamma)=\lim _{u \rightarrow 0} \varepsilon(\alpha-R(u) \mid u, \gamma)
$$

solves the integral equation

$$
\begin{aligned}
& \varepsilon_{k}(\alpha \mid \gamma)=e^{\alpha}-\int_{-\infty}^{\infty} d \alpha^{\prime} G\left(\alpha-\alpha^{\prime} \mid \gamma\right) \ln \left[1+e^{-\varepsilon_{k}\left(\alpha^{\prime} \mid \gamma\right)}\right]+ \\
& \int_{-\infty}^{\infty} d \alpha^{\prime} G\left(\alpha-\alpha^{\prime}+i \pi-i 0 \mid \gamma\right) \ln \left[1+e^{-\bar{\varepsilon}_{k}\left(\alpha^{\prime} \mid \gamma\right)}\right]
\end{aligned}
$$

The function $\eta(\alpha \mid u, \gamma)$ was treated as a small correcting term vanishing at $u \rightarrow 0$.

A similar representation was used in [15] for the logarithm function

$$
L(\alpha \mid u, \gamma)=L_{k}(\alpha-R(u) \mid \gamma)+L_{k}(-\alpha-R(u) \mid \gamma)-\ln 2+l(\alpha \mid u, \gamma),
$$

where

$$
L(\alpha \mid u, \gamma)=\ln \left[1+e^{-\varepsilon(\alpha \mid u, \gamma)}\right], \quad L_{k}(\alpha \mid \gamma)=\ln \left[1+e^{-\varepsilon_{k}(\alpha \mid \gamma)}\right],
$$

and $l(\alpha \mid u, \gamma)$ was also supposed to vanish at $u \rightarrow 0$. Substitution of (31) into 25) leads after some manipulations to the following representation of the Casimir scaling function:

$$
\begin{aligned}
& Y(u \mid \gamma)=-\frac{1}{2 \pi} \int_{-\infty}^{\infty} d \alpha e^{\alpha} \operatorname{Re} L_{k}(\alpha \mid \gamma)- \\
& \frac{u^{2}}{2 \pi} \int_{-\infty}^{\infty} d \alpha e^{-\alpha} \operatorname{Re} \partial_{\alpha} L_{k}(\alpha \mid \gamma)-\frac{u}{\pi} \int_{-\infty}^{\infty} d \alpha \cosh \alpha \operatorname{Re} l(\alpha \mid u, \gamma)
\end{aligned}
$$

The first term in the right-hand side calculated by means of the well-known dilogarithmfunction trick gives the CFT-predicted value,

$$
-\frac{1}{2 \pi} \int_{-\infty}^{\infty} d \alpha e^{\alpha} \operatorname{Re} L_{k}(\alpha \mid \gamma)=-\frac{\pi}{6}
$$

In order to compute the second term in the right-hand side of (33), the following arguments were adopted. To provide convergency of the second integral in the righthand side of (33) at $\alpha \rightarrow-\infty$, the function $\varepsilon_{k}(\alpha \mid \gamma)$ must decay faster than $e^{\alpha}$. After differentiation of equation 30 , one obtains:

$$
\begin{aligned}
& \partial_{\alpha} \varepsilon_{k}(\alpha \mid \gamma)=e^{\alpha}-\int_{-\infty}^{\infty} d \alpha^{\prime} G\left(\alpha-\alpha^{\prime} \mid \gamma\right) \partial_{\alpha^{\prime}} L_{k}\left(\alpha^{\prime} \mid \gamma\right)+ \\
& \int_{-\infty}^{\infty} d \alpha^{\prime} G\left(\alpha-\alpha^{\prime}+i \pi-i 0 \mid \gamma\right) \partial_{\alpha^{\prime}} \overline{L_{k}\left(\alpha^{\prime} \mid \gamma\right)}
\end{aligned}
$$

Then adopting the asymptotic expansion of the kernel $G(\alpha \mid \gamma)$ at large $|\alpha|$

$$
G(\alpha \mid \gamma)=\mathfrak{a}_{1} e^{-|\alpha|}+\mathfrak{b}_{1} e^{-2 \hat{\gamma}|\alpha| / \pi}+\ldots,
$$


where $\hat{\gamma}=\gamma(1-\gamma / \pi)^{-1}$ and

$$
\mathfrak{a}_{1}=\frac{1}{\pi} \tan \left(\frac{\pi^{2}}{2 \gamma}\right), \quad \mathfrak{b}_{1}=\frac{\hat{\gamma}}{\pi^{2}} \tan \left(\frac{\pi^{2}}{\pi-\gamma}\right),
$$

and proceeding to the limit $\alpha \rightarrow-\infty$ in equation (34), Destri and de Vega obtained

$\partial_{\alpha} \varepsilon_{k}(\alpha \mid \gamma) \simeq\left[1-2 \mathfrak{a}_{1} \int_{-\infty}^{\infty} d \alpha^{\prime} e^{-\alpha^{\prime}} \operatorname{Re} \partial_{\alpha^{\prime}} L_{k}\left(\alpha^{\prime} \mid \gamma\right)\right] e^{\alpha}+O\left(e^{2 \alpha}\right)+O\left(e^{2 \hat{\gamma} \alpha / \pi}\right)$.

Putting the coefficient of $\left(e^{\alpha}\right)$ to zero, they conclude that

$$
\int_{-\infty}^{\infty} d \alpha^{\prime} e^{-\alpha^{\prime}} \operatorname{Re} \partial_{\alpha^{\prime}} L_{k}\left(\alpha^{\prime} \mid \gamma\right)=\frac{1}{2 \mathfrak{a}_{1}}
$$

and

$$
Y(u \mid \gamma)=-\frac{\pi}{6}-\frac{u^{2}}{4} \cot \frac{\pi^{2}}{2 \gamma}-\frac{u}{\pi} \int_{-\infty}^{\infty} d \alpha \cosh \alpha \operatorname{Re} l(\alpha \mid u, \gamma)
$$

The small- $u$ asymptotics of the third term in the right-hand side of (38) was studied in Section 7.4 of [15]. Destri and de Vega came there to the conclusion, that this term admits at $u \rightarrow 0$ the asymptotical expansion of the form

$$
-\frac{u}{\pi} \int_{-\infty}^{\infty} d \alpha \cosh \alpha \operatorname{Re} l(\alpha \mid u, \gamma)=\sum_{n=0}^{\infty} C_{n}(\gamma) u^{4 n \gamma / \pi}
$$

So, the final result of [15] for the ultraviolet asymptotics $u \rightarrow 0$ of the Casimir scaling function in the sine-Gordon model in the repulsive regime takes the form:

$$
Y(u \mid \gamma)=-\frac{\pi}{6}-\frac{u^{2}}{4} \cot \frac{\pi^{2}}{2 \gamma}+\sum_{n=0}^{\infty} C_{n}(\gamma) u^{4 n \gamma / \pi}
$$

No explicit expressions for the coefficients $C_{n}(\gamma)$ were given in [15].

It turns out, however, that the derivation of (40) given in [15] and sketched above is inconsistent and contains several mistakes. First, equation (28) representing the pseudoenergy $\varepsilon(\alpha \mid u, \gamma)$ can be useful in the asymptotical analysis, if two initial terms in its right-hand side dominate in the limit $u \rightarrow 0$, while the term $\eta(\alpha \mid u, \gamma)$ describes the small correction. However, this is not the case, since the pseudoenergy $\varepsilon(\alpha \mid u, \gamma)$ is the complex-valued function having the reflection symmetry

$$
\varepsilon(\alpha \mid u, \gamma)=\overline{\varepsilon(-\alpha \mid u, \gamma)}
$$

which is not respected by the function $\varepsilon_{k}(\alpha-R(u) \mid \gamma)+\varepsilon_{k}(-\alpha-R(u) \mid \gamma)$ in the right-hand side of (28). So, instead of (28), one should write

$$
\varepsilon(\alpha \mid u, \gamma)=\varepsilon_{k}(\alpha-R(u) \mid \gamma)+\bar{\varepsilon}_{k}(-\alpha-R(u) \mid \gamma)+\eta(\alpha \mid u, \gamma)
$$

Equation (31) for the logarithm functions requires the same correction:

$$
L(\alpha \mid u, \gamma)=L_{k}(\alpha-R(u) \mid \gamma)+\bar{L}_{k}(-\alpha-R(u) \mid \gamma)-\ln 2+l(\alpha \mid u, \gamma),
$$

where $\bar{L}_{k}(-\alpha-R(u) \mid \gamma)=\overline{L_{k}(-\alpha-R(u) \mid \gamma)}$.

There is, however, one more problem with equation (31), which remains also in its improved version 43 . Its zero-order part

$$
L^{(0)}(\alpha \mid u, \gamma)=L_{k}(\alpha-R(u) \mid \gamma)+\bar{L}_{k}(-\alpha-R(u) \mid \gamma)-\ln 2
$$


approximates well at small $u$ the exact function $L(\alpha \mid u, \gamma)$ in the interval $-R(u)<\alpha<$ $R(u)$, but is not appropriate at larger $|\alpha|>R(u)$. Really, one can easily see that the pseudoenergy $\varepsilon(\alpha \mid u, \gamma)$ exponentially increases at large $|\alpha| \rightarrow \infty$, namely

$$
\varepsilon(\alpha \mid u, \gamma) \simeq e^{|\alpha|-R(u)} \quad \text { at }|\alpha|-R(u) \gg 1 .
$$

Accordingly, the difference $L(\alpha \mid u, \gamma)-\ln 2$ decays extremely fast at large $|\alpha|$, as

$$
L(\alpha \mid u, \gamma)-\ln 2=-\frac{1}{2} \exp \left[-e^{|\alpha|-R(u)}\right]+\ldots, \quad \text { at }|\alpha|-R(u) \gg 1 .
$$

In contrast, the difference $L^{(0)}(\alpha \mid u, \gamma)-\ln 2$ decays much slower at $|\alpha| \rightarrow \infty$. Really, if we assume that $\varepsilon_{k}(\alpha \mid \gamma)$ exponentially decays at large negative $\alpha$,

$$
\varepsilon_{k}(\alpha \mid \gamma) \simeq \frac{b(\gamma)}{a(\gamma)} e^{a(\gamma) \alpha} \quad \text { at } \alpha \rightarrow-\infty
$$

with some $a(\gamma)>0$, we get

$$
L^{(0)}(\alpha \mid u, \gamma)-\ln 2=-\frac{\overline{b(\gamma)}}{2 a(\gamma)} e^{-a(\gamma)[\alpha+R(u)]}+\ldots, \quad \text { at } \alpha-R(u) \gg 1,
$$

instead of 45).

We shall see in the next Section that assumption (46) is indeed correct, and that

$$
a(\gamma)=\frac{2 \gamma}{\pi}
$$

Therefore, the integrand in the second integral in the right-hand side of (33) behaves at $\alpha \rightarrow-\infty$ as

$$
e^{-\alpha} \operatorname{Re} \partial_{\alpha} L_{k}(\alpha \mid \gamma) \simeq-\frac{\operatorname{Re} b(\gamma)}{2} \exp \left[\left(\frac{2 \gamma}{\pi}-1\right) \alpha\right]
$$

Since $\left(\frac{2 \gamma}{\pi}-1\right)<0$ in the repulsive case $0<\gamma<\pi / 2$, the integrand 48 ) exponentially increases at $\alpha \rightarrow-\infty$, and the second integral in the right-hand side of (33) diverges at $\alpha \rightarrow-\infty$. Therefore, the very assumptions of Destri and de Vega about convergency of that integral is not valid, and their subsequent analysis based on this assumption is not satisfactory.

In the next Section, we present the consistent derivation of the small- $u$ asymptotic expansion for the Casimir scaling function $Y(u \mid \gamma)$, which is free from the problems outlined above.

\section{Ultraviolet asymptotics of the Casimir scaling function}

Before proceeding to the calculations, let us summarise the obtained results. For generic values of $\gamma \in(0, \pi)$, the Casimir scaling function $Y(u \mid \gamma)$ has the small- $u$ asymptotical expansion of the form $(40)$ predicted by Destri and de Vega, with $C_{0}(\gamma) \equiv 0$, and $C_{1}(\gamma)$ is given by equation (4). The asymptotic formula

$$
Y(u \mid \gamma)+\frac{\pi}{6}=C_{1}(\gamma) u^{4 \gamma / \pi}-\frac{u^{2}}{4} \cot \left(\frac{\pi^{2}}{2 \gamma}\right)+\sum_{n=2}^{\infty} C_{n}(\gamma) u^{4 n \gamma / \pi}
$$




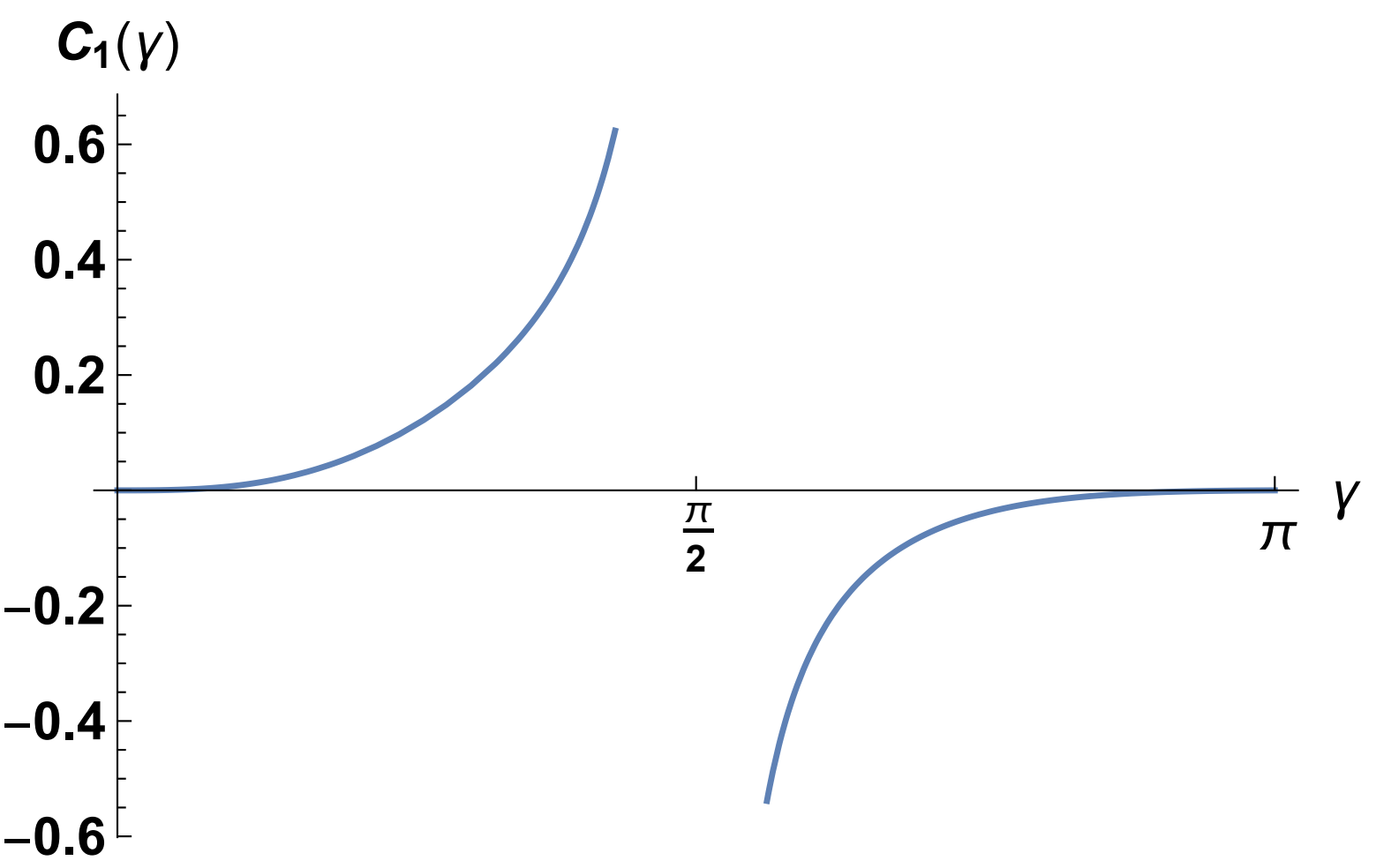

Figure 1: Plot of the coefficient $C_{1}(\gamma)$ defined by (4).

holds at $u \rightarrow 0$ both in the repulsive and attractive cases of the sine-Gordon model for all values of the parameter $\gamma$ in the interval $0<\gamma<\pi$, apart from the points $\gamma_{l}=\frac{\pi}{2 l}$, with $l=1,2, \ldots$.

The first term in the right-hand side of 49 dominates over the second one in the repulsive regime $0<\gamma<\pi / 2$. In the attractive regime $\pi / 2<\gamma<\pi$, the second term becomes larger than the first one. Plot of the coefficient $C_{1}(\gamma)$ defined by (4) is shown in Figure 1. This function has a simple pole in the free-fermionic point $\gamma=\pi / 2$. At this point, formula (49) should be replaced by

$$
Y(u \mid \pi / 2)+\frac{\pi}{6}=\frac{u^{2}}{2 \pi}\left(-\ln \frac{u}{\pi}+\frac{1}{2}-\gamma_{E}\right)+O\left(u^{4}\right)
$$

where $\gamma_{E}$ is the Euler's constant. At the points $\gamma_{l}=\frac{\pi}{2 l}$ with $l=2,3, \ldots$, equation 49. is to be replaced by

$$
Y\left(u \mid \gamma_{l}\right)+\frac{\pi}{6}=C_{1}\left(\gamma_{l}\right) u^{2 / l}-\frac{u^{2}}{2 \pi l}[\ln u+O(1)]+O\left(u^{4 / l}\right) .
$$

Proceeding to derivation of these results, we start from the conformal limit $u=0$ in the repulsive case $0<\gamma<\pi / 2$, and rewrite equation (34) in the form

$$
\begin{aligned}
& \partial_{\alpha} \varepsilon_{k}(\alpha \mid \gamma)=e^{\alpha}-2 \int_{-\infty}^{\infty} d \alpha^{\prime} G\left(\alpha-\alpha^{\prime} \mid \gamma\right) \operatorname{Re} \partial_{\alpha^{\prime}} L_{k}\left(\alpha^{\prime} \mid \gamma\right)- \\
& \int_{-\infty}^{\infty} d \alpha^{\prime} g\left(\alpha-\alpha^{\prime}-i 0 \mid \gamma\right) \partial_{\alpha^{\prime}} \overline{L_{k}\left(\alpha^{\prime} \mid \gamma\right)}
\end{aligned}
$$

using equality (23). We suppose, that the pseudoenergy $\varepsilon_{k}(\alpha \mid \gamma)$ corresponding to the critical point $u=0$ exponentially decays at large negative $\alpha$ according to (46), with 
some positive exponent $0<a(\gamma)<1$. Then we proceed to the limit of a large negative $\alpha$, replace the derivative of the logarithm function $L_{k}\left(\alpha^{\prime} \mid \gamma\right)$ defined by (32) by the first term in its Taylor expansion,

$$
\partial_{\alpha^{\prime}} L_{k}\left(\alpha^{\prime} \mid \gamma\right)=-\frac{\partial_{\alpha^{\prime}} \varepsilon_{k}\left(\alpha^{\prime} \mid \gamma\right)}{2}+\ldots
$$

and neglect the term $e^{\alpha}$ in the right-hand-side of equation (52). As the result, we obtain the linear uniform integral equation

$$
\begin{aligned}
& \partial_{\alpha} \varepsilon_{k}(\alpha \mid \gamma)=\int_{-\infty}^{\infty} d \alpha^{\prime} G\left(\alpha-\alpha^{\prime} \mid \gamma\right) \operatorname{Re} \partial_{\alpha^{\prime}} \varepsilon_{k}\left(\alpha^{\prime} \mid \gamma\right)+ \\
& \frac{1}{2} \int_{-\infty}^{\infty} d \alpha^{\prime} g\left(\alpha-\alpha^{\prime}-i 0 \mid \gamma\right) \partial_{\alpha^{\prime}} \overline{\varepsilon_{k}\left(\alpha^{\prime} \mid \gamma\right)}
\end{aligned}
$$

which determines the asymptotics of the pseudoenergy $\varepsilon_{k}(\alpha \mid \gamma)$ at $\alpha \rightarrow-\infty$, up to a real numerical factor. The solution of this equation indeed has the form consistent with (46), namely

$$
\begin{aligned}
& \partial_{\alpha} \varepsilon_{k}(\alpha \mid \gamma)=b e^{2 \alpha \gamma / \pi} \\
& b=-i c_{1}(\gamma) e^{i \gamma}
\end{aligned}
$$

The real numerical factor $c_{1}(\gamma)$ is in fact positive. It will be determined later, see equation (66). In deriving (54a), (54b) we have used two explicit integral formulas, which are valid at $0<\gamma<\pi / 2$ and $0<a<\min (1, \hat{\gamma})$,

$$
\begin{aligned}
& \int_{-\infty}^{\infty} d \alpha e^{a \alpha} g(-\alpha-i 0 \mid \gamma)=-e^{\pi i a / 2} \frac{\sin \left[\pi a\left(\nu^{-1}-2^{-1}\right)\right]}{\sin (\pi a / \nu)} \\
& \int_{-\infty}^{\infty} d \alpha e^{a \alpha} G(-\alpha \mid \gamma)=\frac{\sin \left[\pi a\left(\nu^{-1}-2^{-1}\right)\right]}{2 \cos (\pi a / 2) \sin (\pi a / \nu)}
\end{aligned}
$$

where $\nu=\frac{2 \gamma}{\pi-\gamma}$.

Let us turn now to the asymptotical behaviour of the pseudoenergy function $\varepsilon(\alpha \mid u, \gamma)$ at a small nonzero scaling parameter $u \ll 1$. In this limit, one can neglect to the leading order in $u$ the term $\eta(\alpha \mid u, \gamma)$ in equation 42 and represent this function as

$$
\varepsilon(\alpha \mid u, \gamma) \approx \varepsilon_{k}(\alpha-R(u) \mid \gamma)+\bar{\varepsilon}_{k}(-\alpha-R(u) \mid \gamma)
$$

At $|\alpha| \ll R(u)$, we can use for the function $\varepsilon_{k}(\alpha \mid \gamma)$ the asymptotical formula (46), and reduce (56) to the form

$$
\varepsilon(\alpha \mid u, \gamma) \approx\left(\frac{u}{2}\right)^{a(\gamma)} \frac{b e^{a(\gamma) \alpha}+\bar{b} e^{-a(\gamma) \alpha}}{a(\gamma)},
$$

with $a(\gamma)$ given by 47$)$. Combining this with (54b), one finds,

$$
\begin{aligned}
& \operatorname{Re} \varepsilon(\alpha \mid u, \gamma) \approx\left(\frac{u}{2}\right)^{2 \gamma / \pi} \frac{2 c_{1}(\gamma) \cosh (2 \gamma \alpha / \pi)}{a(\gamma)} \sin \gamma \\
& \operatorname{Im} \varepsilon(\alpha \mid u, \gamma) \approx-\left(\frac{u}{2}\right)^{2 \gamma / \pi} \frac{2 c_{1}(\gamma) \sinh (2 \gamma \alpha / \pi)}{a(\gamma)} \cos \gamma
\end{aligned}
$$


Thus, the ratios $\operatorname{Re} \varepsilon(\alpha \mid u, \gamma) / \cosh (2 \gamma \alpha / \pi)$ and $\operatorname{Im} \varepsilon(\alpha \mid u, \gamma) / \sinh (2 \gamma \alpha / \pi)$ must have at $u \ll 1$ wide plateaus near the origin $\alpha=0$ at the values

$$
\begin{aligned}
& \frac{\operatorname{Re} \varepsilon(0 \mid u, \gamma)}{\cosh (2 \gamma \alpha / \pi)} \approx\left(\frac{u}{2}\right)^{2 \gamma / \pi} \frac{\pi c_{1}(\gamma)}{\gamma} \sin \gamma, \\
& \frac{\operatorname{Im} \varepsilon(0 \mid u, \gamma)}{\sinh (2 \gamma \alpha / \pi)} \approx-\left(\frac{u}{2}\right)^{2 \gamma / \pi} \frac{\pi c_{1}(\gamma)}{\gamma} \cos \gamma
\end{aligned}
$$

and

$$
\lim _{u \rightarrow 0} \lim _{\alpha \rightarrow 0} \frac{\operatorname{Re} \varepsilon(\alpha \mid u, \gamma)}{\cosh (2 \gamma \alpha / \pi)} / \frac{\operatorname{Im} \varepsilon(\alpha \mid u, \gamma)}{\sinh (2 \gamma \alpha / \pi)}=-\tan \gamma
$$

These properties of the pseudoenergy $\varepsilon(\alpha \mid u, \gamma)$ at a small $u$ in the repulsive regime $0<\gamma<\pi / 2$ were checked in numerical calculations described in the next Section, see Figure 4 .

The outlined above perturbative analysis of the integral equation (52) at $\alpha \rightarrow-\infty$ can be extended to higher orders in $\exp (2 \alpha \gamma / \pi)$. It leads to the following asymptotic expansion for the pseudoenergy $\varepsilon_{k}(\alpha \mid \gamma)$ at $\alpha \rightarrow-\infty$ :

$$
\varepsilon_{k}(\alpha \mid \gamma)=-\frac{i \pi}{2 \gamma} \sum_{n=1}^{\infty} \frac{c_{n}(\gamma)}{n} \exp \left[\frac{2 n \gamma}{\pi}\left(\alpha+\frac{i \pi}{2}\right)\right],
$$

with real coefficients $c_{n}(\gamma)$. Note, that the function

$$
\mathfrak{f}_{k}(\alpha \mid \gamma)=\exp \left[-\varepsilon_{k}(\alpha-i \pi / 2 \mid \gamma)\right]
$$

solves the "massless" version of the DDV equation:

$$
-i \ln \mathfrak{f}_{k}(\alpha \mid \gamma)=e^{\alpha}+2 \int_{-\infty}^{\infty} d \alpha^{\prime} G\left(\alpha-\alpha^{\prime} \mid \gamma\right) \operatorname{Im} \ln \left[1+\mathfrak{f}_{k}\left(\alpha^{\prime}+i 0 \mid \gamma\right)\right]
$$

The "counting function" $\phi_{k}(\alpha \mid \gamma)=-i \ln \mathfrak{f}_{k}(\alpha \mid \gamma)$ in the left-hand side of this equation is real at real $\alpha$. Its asymptotic expansion at $\alpha \rightarrow-\infty$ can be read from (61), 62):

$$
\phi_{k}(\alpha \mid \gamma)=\frac{\pi}{2 \gamma} \sum_{n=1}^{\infty} \frac{c_{n}(\gamma)}{n} e^{2 n \gamma \alpha / \pi}
$$

It was shown in [4], that the same expansion for this counting function holds also in the attractive case $\pi / 2<\gamma<\pi$, see the discussion below in Appendix C.

Now let us return to the asymptotic expansion of the Casimir scaling functions $Y(u \mid \gamma)$ in the ultraviolet limit $u \rightarrow 0$. By means of the perturbative solution of the TBA equations (26), we obtained at $0<\gamma<\pi / 2$ the first sub-leading term in this expansion:

$$
Y(u \mid \gamma)+\frac{\pi}{6}=\frac{\pi^{2}\left[c_{1}(\gamma)\right]^{2}}{4 \gamma^{2} \sin (2 \gamma)}(u / 2)^{4 \gamma / \pi}+\ldots
$$

Derivation of this asymptotic formula is described in Appendix A. The coefficient $c_{1}(\gamma)$ in (65) is the same as in expansions (61), (64). It turns out, that the direct calculation of the constant $c_{1}(\gamma)$ from equation 30 presents a difficult problem. By this reason, we determined the coefficient $C_{1}(\gamma)$ in a completely different way exploiting the perturbative CFT technique. This calculation is described in Appendix B, and the 


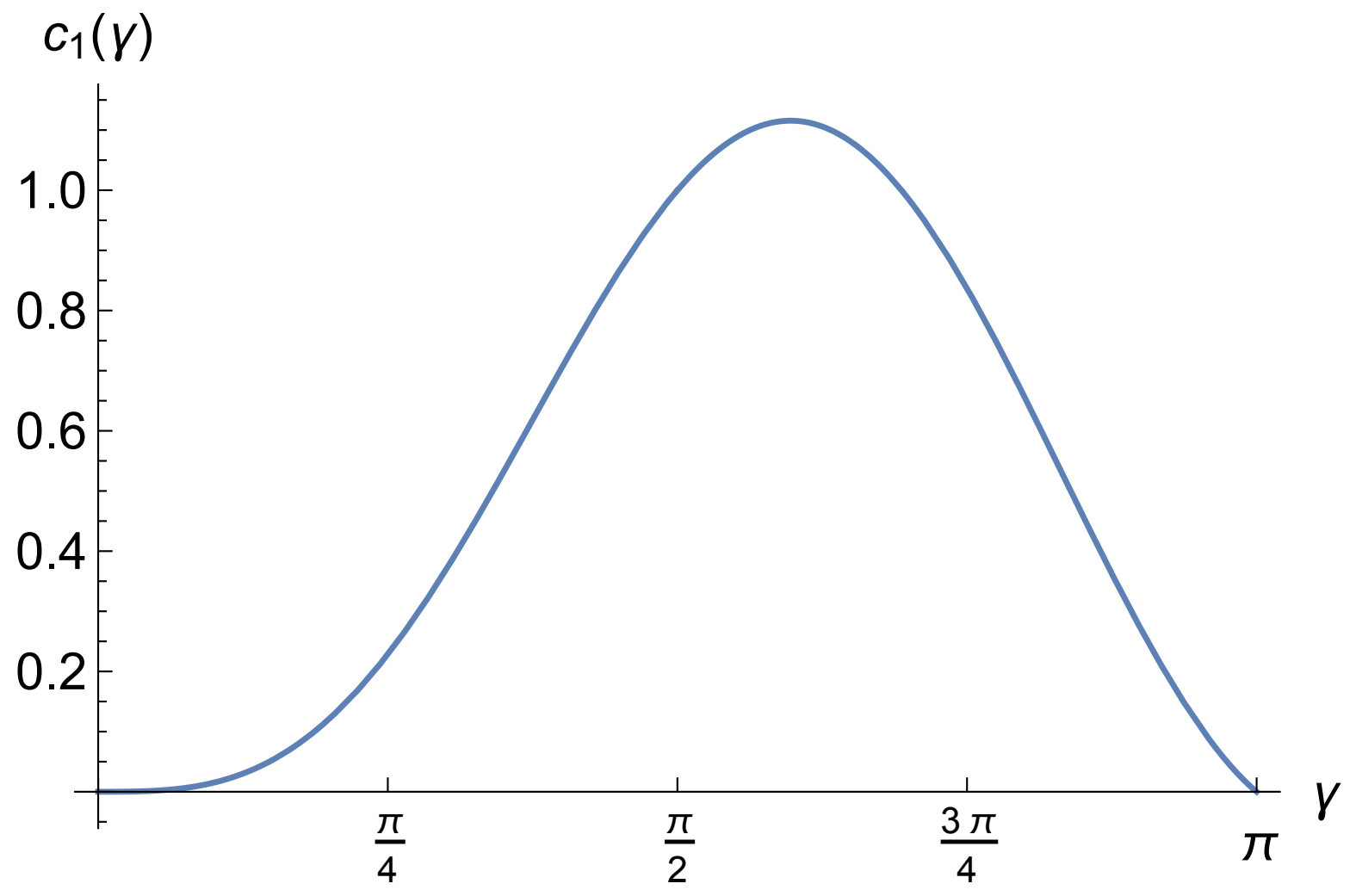

Figure 2: The coefficient $c_{1}(\gamma)$ determined by equation 66 .

resulting expression for the coefficient $C_{1}(\gamma)$ is given in equation (4). Using this result, one can recover $\ddagger$ the coefficient $c_{1}(\gamma)$ from equations (49), 65):

$$
\begin{aligned}
& c_{1}(\gamma)=4 \pi^{3 / 2} \frac{\gamma \cot \gamma}{\pi-2 \gamma} \frac{\Gamma\left(\frac{1}{2}+\frac{\gamma}{\pi}\right)}{\left[\Gamma\left(\frac{\gamma}{\pi}\right)\right]^{3}}\left[\frac{\Gamma\left(\frac{\pi}{2 \gamma}\right)}{\sqrt{\pi} \Gamma\left(-\frac{1}{2}+\frac{\pi}{2 \gamma}\right)}\right]^{2 \gamma / \pi}= \\
& \left.4 \pi^{3 / 2}\left(\frac{2}{\pi}\right)^{2 \gamma / \pi} \frac{\gamma \cos \gamma \Gamma\left(\frac{1}{2}+\frac{\gamma}{\pi}\right)}{(\pi-2 \gamma) \Gamma\left(\frac{\gamma}{\pi}\right)} \kappa(\xi)\right|_{\xi=(\pi-\gamma) / \gamma} .
\end{aligned}
$$

Plot of this function is shown in Figure 2. The function $c_{1}(\gamma)$ is analytical in the whole interval $0<\gamma<\pi$, and has the essential singularity at $\gamma=0$.

Though our derivation of formula (66) for the coefficient $c_{1}(\gamma)$ was limited to the repulsive case $0<\gamma<\pi / 2$, it remains valid in the attractive regime $\pi / 2<\gamma<\pi$ as well. In the latter case, the TBA equation in the form 30 does not hold any more, and one should define the coefficients $c_{n}(\gamma)$ from the expansion (64) for the solution of the DDV equation (63). This integral equation was studied in the attractive regime $\pi / 2<\gamma<\pi$ to much details by Bazhanov, Lukyanov, and Zamolodchikov [4]. It turns out, that the explicit expression for the coefficient $c_{1}(\gamma)$ in the attractive regime, which can be gained from [4], coincides exactly with our result (66), analytically continued into the interval $\pi / 2<\gamma<\pi$. The details are given in Appendix C, were we recall ¥ In fact, equation 65 determines $c_{1}(\gamma)$ up to the sign. The latter is fixed by the known value $c_{1}(\pi / 2)=1$ at the free-fermionic point $\gamma=\pi / 2$, where $\phi_{k}(\alpha \mid \pi / 2)=e^{\alpha}$. 


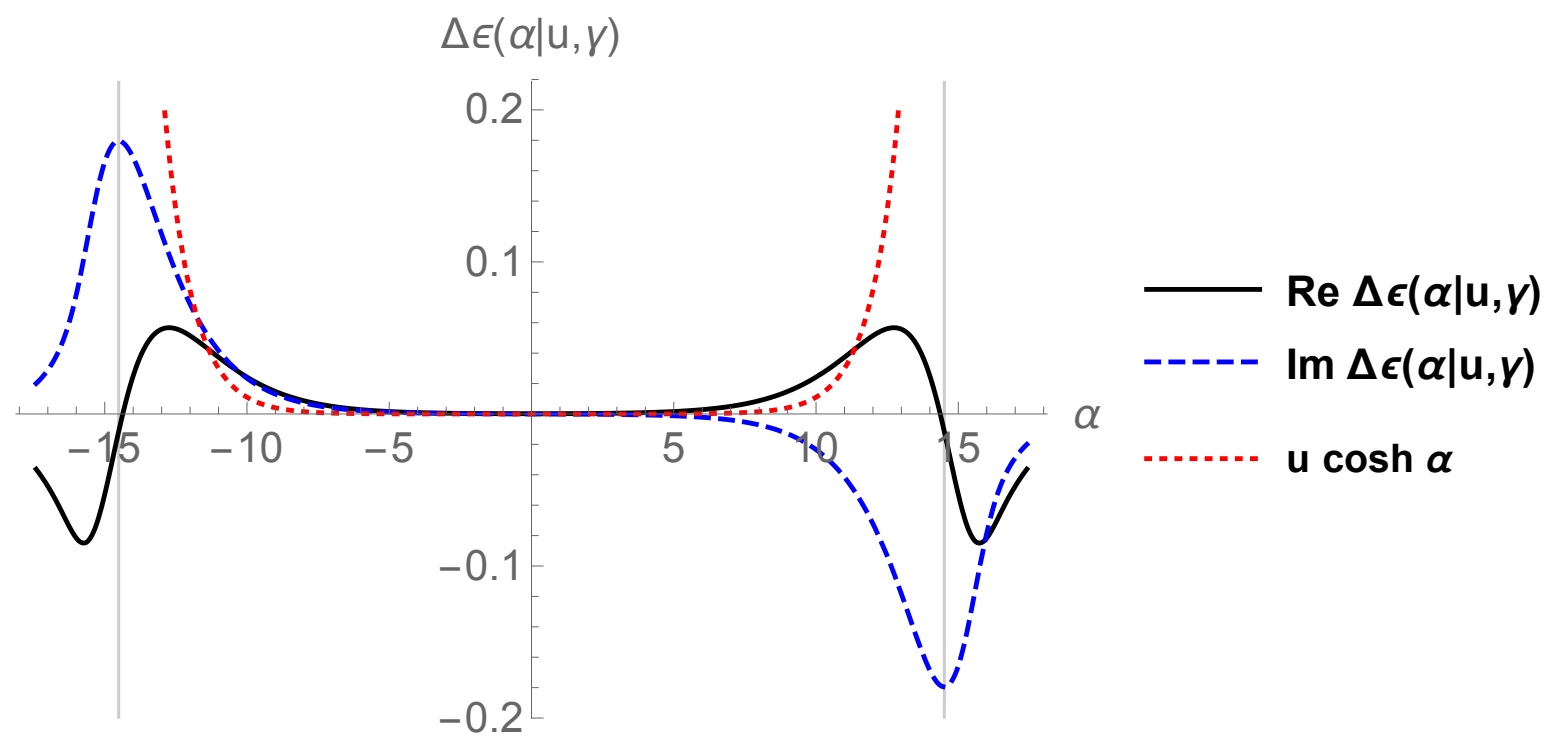

Figure 3: Real (solid black) and imaginary (dashed blue) parts of the function $\Delta \varepsilon(\alpha \mid u, \gamma)$ defined by 70 versus rapidity $\alpha$ at $\gamma=0.3 \pi$ and $u=10^{-6}$. Vertical lines are located at $\pm R(u)$, with $R(u) \approx 14.5$.

some of results of the work [4].

\section{Numerical work}

The results described in the previous Section were confirmed by numerical calculations both in the repulsive and attractive regimes.

In the repulsive regime $0<\gamma<\pi / 2$, we calculated the pseudoenergy $\varepsilon(\alpha \mid u, \gamma)$ by iterative solution of the nonlinear TBA equations (26), and then obtained the Casimir scaling function $Y(u \mid \gamma)$ using the integral formula (25).

In the attractive regime $\pi / 2<\gamma<\pi$, the nonlinear integral equation (21) is still valid, but the TBA equations in the form (26) do not hold any more. The reason is that the integral kernel $G(\alpha \mid \gamma)$ defined by $22 p$ has the $t$-channel poles at $\alpha_{n}=i n \pi\left(\frac{\pi}{\gamma}-1\right)$, with $n=1,2 \ldots$ In the attractive regime, one or several such poles come into the physical strip $0<\operatorname{Im} \alpha<\pi$ indicating appearance of the soliton-antisoliton bound states in the particle spectrum $\{$.

These poles prevent analytical continuation of equation (21) from the real $\alpha$-axis into the lines $\operatorname{Im} \alpha= \pm \pi / 2$, which has been used in derivation of the TBA equation (26) in the repulsive case. To avoid this problem, we have used for numerical calculations in the attractive case the modified TBA equations, which were obtained from (21) by analytical continuation in the rapidity variable $\alpha$ from the real axis into the line $\S$ These bound states have the masses [24] $m_{n}=2 m \sin (\pi n \xi / 2)=2 m \cosh \left[\left(i \pi-\alpha_{n}\right) / 2\right]$. 
$\operatorname{Im} \alpha=\delta$, with some $\delta$ lying in the interval

$$
\delta \in\left(0, \frac{\pi(\pi-\gamma)}{2 \gamma}\right) .
$$

These modified TBA equations read as,

$$
\begin{aligned}
& \varepsilon(\alpha \mid u, \gamma, \delta)=-i u \sinh (\alpha+i \delta)-\int_{-\infty}^{\infty} d \alpha^{\prime} G\left(\alpha-\alpha^{\prime} \mid \gamma\right) \ln \left[1+e^{-\varepsilon\left(\alpha^{\prime} \mid u, \gamma, \delta\right)}\right] \\
& +\int_{-\infty}^{\infty} d \alpha^{\prime} G\left(\alpha-\alpha^{\prime}+2 i \delta\right) \ln \left[1+e^{-\bar{\varepsilon}\left(\alpha^{\prime} \mid u, \gamma, \delta\right)}\right] \\
& \bar{\varepsilon}(\alpha \mid u, \gamma, \delta)=i u \sinh (\alpha-i \delta)-\int_{-\infty}^{\infty} d \alpha^{\prime} G\left(\alpha-\alpha^{\prime} \mid \gamma\right) \ln \left[1+e^{-\bar{\varepsilon}\left(\alpha^{\prime} \mid u, \gamma, \delta\right)}\right] \\
& +\int_{-\infty}^{\infty} d \alpha^{\prime} G\left(\alpha-\alpha^{\prime}-2 i \delta\right) \ln \left[1+e^{-\varepsilon\left(\alpha^{\prime} \mid u, \gamma, \delta\right)}\right]
\end{aligned}
$$

where

$$
\varepsilon(\alpha \mid u, \gamma, \delta)=-\ln f(\alpha+i \delta \mid u, \gamma)
$$

is the modified pseudoenergy, and $\bar{\varepsilon}(\alpha \mid u, \gamma, \delta)$ is its complex conjugate,

$$
\bar{\varepsilon}(\alpha \mid u, \gamma, \delta)=-\overline{\ln f(\alpha+i \delta \mid u, \gamma)}=\ln f(\alpha-i \delta \mid u, \gamma) .
$$

The Casimir scaling function $Y(u \mid \gamma)$ can be expressed in terms of the modified pseudoenergy as follows,

$$
Y(u \mid \gamma)=-\frac{u}{\pi} \operatorname{Im} \int_{-\infty}^{\infty} d \alpha \sinh (\alpha+i \delta) \ln \left[1+e^{-\varepsilon(\alpha \mid u, \gamma, \delta)}\right] .
$$

The integral in the right-hand side in fact does not depend on the parameter $\delta$ provided the latter lies in the allowed interval (67).

Figure 3 displays the real and imaginary parts of the difference

$$
\Delta \varepsilon(\alpha \mid u, \gamma)=\varepsilon(\alpha \mid u, \gamma)-u \cosh \alpha
$$

plotted agains the rapidity $\alpha$ in the repulsive regime at $\gamma=0.3 \pi$ and $u=10^{-6}$. It was shown in the previous Section, that the pseudoenergy $\varepsilon_{k}(\alpha \mid \gamma)$ corresponding to the critical point $u=0$ decays at $\alpha \rightarrow-\infty$ as $\sim e^{2 \gamma \alpha / \pi}$. Despite the claim of Destri and de Vega in [15], this exponential decay is slower than $e^{\alpha}$ in the repulsive regime $0<\gamma<\pi / 2$. By this reason, and due to the asymptotical formulas (56), (57), the first term in the right-hand side of $(70)$ dominates over the second one at $R(u)-|\alpha| \gg 1$. Therefore,

$$
|\Delta \varepsilon(\alpha \mid u, \gamma)| \gg u \cosh \alpha
$$

in this region of the rapidity variable $\alpha$. As one can see in Figure 3 , the above strong inequality really holds at $|\alpha| \lesssim 10$ for the chosen values of parameters $\gamma, u$.

Figure 4 provides a more detailed numerical check of the small- $u$ asymptotical formulae (57), 47), (58), and (60) for the pseudoenergy $\varepsilon(\alpha \mid u, \gamma)$ at $\gamma=$ $0.3 \pi$, and $u=10^{-6}$. The solid black and the dashed blue lines display the numerically obtained $\alpha$-dependences of the functions $\operatorname{Re} \varepsilon(\alpha \mid u, \gamma) / \cosh (2 \gamma \alpha / \pi)$ and 


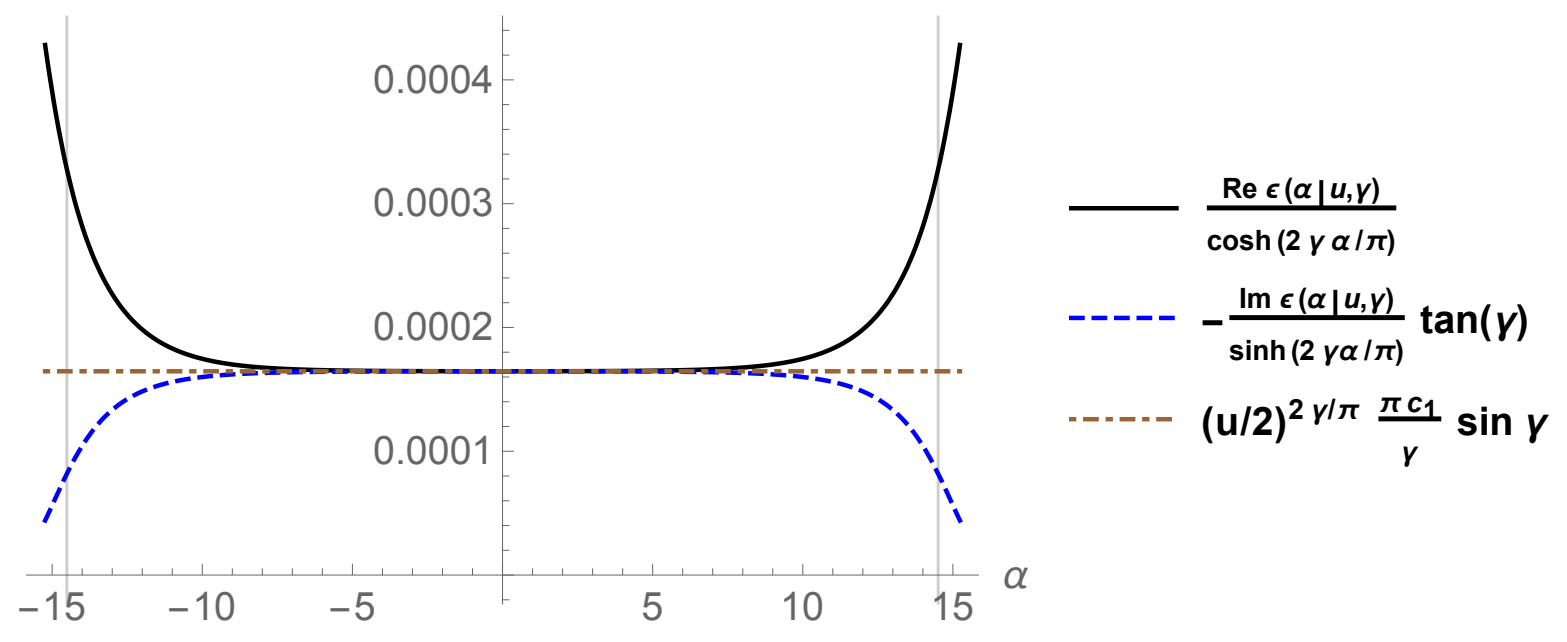

Figure 4: Numerical check of equations (47), 57, 59, ,60, and 66 at $\gamma=0.3 \pi$, $u=10^{-6}$. At a small value of $u$, the functions $\operatorname{Re} \varepsilon(\alpha \mid u, \gamma) / \cosh (2 \gamma \alpha / \pi)$ and $-(\tan \gamma) \operatorname{Im} \varepsilon(\alpha \mid u, \gamma) / \sinh (2 \gamma \alpha / \pi)$ have wide plateaus near the origin $\alpha=0$, where they approach almost the same value. At $u \rightarrow 0$ this value is given by equations (59), (66). Vertical lines are located at $\pm \ln (2 / u)$.

$-(\tan \gamma) \operatorname{Im} \varepsilon(\alpha \mid u, \gamma) / \sinh (2 \gamma \alpha / \pi)$, respectively. In a agreement with the theoretical predictions (58), (60), these two functions display wide plateaus near the origin $\alpha=0$ approaching there to almost the same value. The dot-dashed brown horizontal line represents the theoretical prediction (59) for this value at $u \rightarrow 0$, with the constant $c_{1}(\gamma)$ given by 66 .

Figure 5 summarises the numerical results for the $u$-dependences of the Casimir scaling function $Y(u \mid \gamma)$ at four different values of parameter $\gamma$. The blue solid lines corresponding to the degenerate repulsive regime $\gamma=0$ were plotted using the previously obtained results [6]. The orange dashed lines correspond to the repulsive case at $\gamma=0.3 \pi$. The dashed orange line cannot be distinguished from the solid blue one in Figure 5 a showing the scaling function variation in the wide interval of the scaling parameter $0<u<6$. However, the solid blue and the dashed orange lines are well separated in Figure $5 \mathrm{~b}$ that displays the scaling function variation in the small- $u$ region $0<u<0.1$. The green dot-dashed lines in Figure 5 display the scaling function (B.18) at the free-fermionic point. The red dot-lines in Figure 5 display the Casimir scaling function in the attractive regime $Y(u \mid 0.65 \pi)$.

Figure 6 shows the $u$-dependence of the deviation of the Casimir scaling function $Y(u \mid \gamma)$ from its CFT value $-\pi / 6$ in the repulsive regime at $\gamma=0.3 \pi$ in the small- $u$ regions, $0<u<0.05$ in Figure 6a, and $0<u<0.4$ in Figure 6b. The dots display the numerical data. The dot-dashed blue, dashed orange and solid green lines display one, two and three leading terms, respectively, in the asymptotical formula (49). The coefficient $C_{1}(\gamma)$ is given explicitly by (4). The analytical expression for the coefficient $C_{2}(\gamma)$ is not known. We obtained the numerical value $C_{2}(\gamma=0.3 \pi) \approx-0.13$ of this 

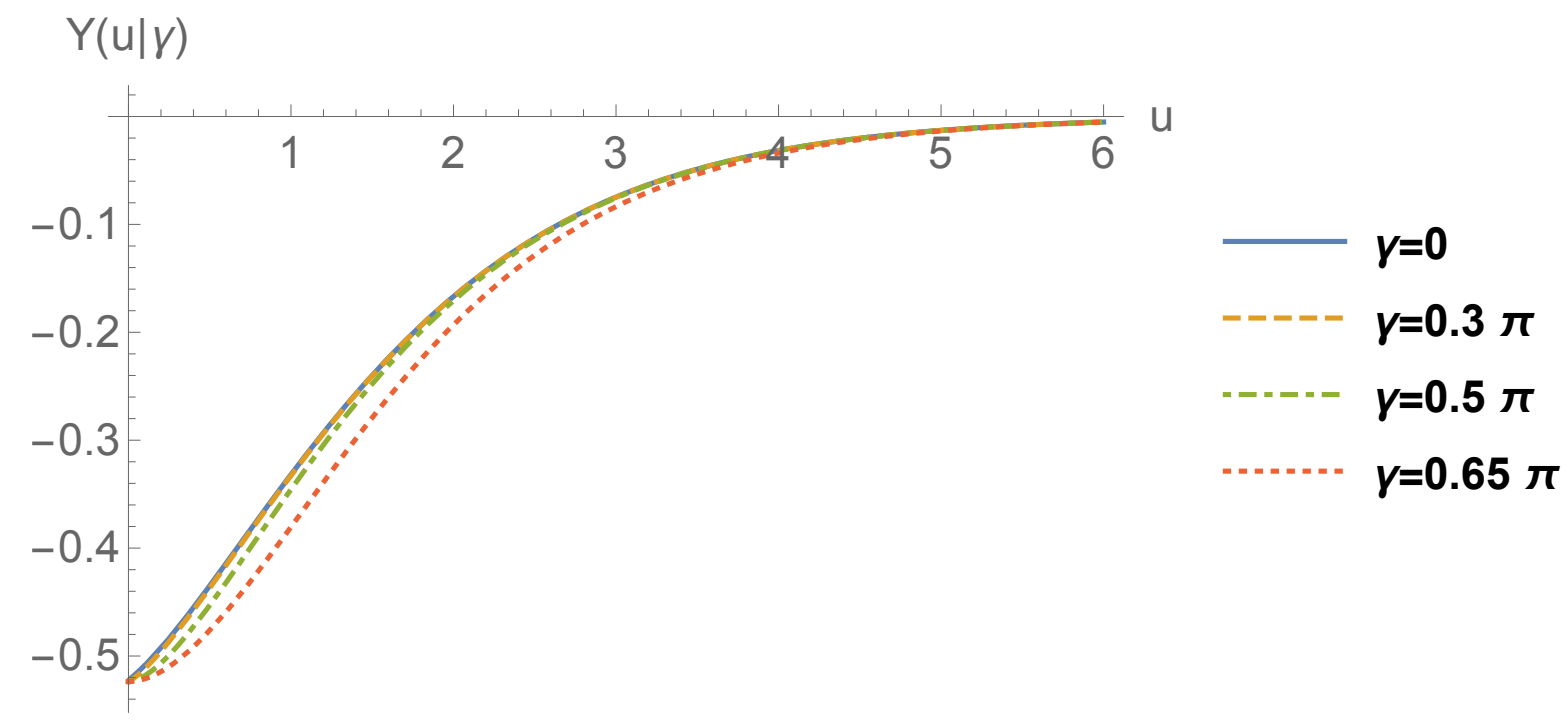

(a)

$\mathrm{Y}(\mathrm{u} \mid \mathrm{\gamma})+\pi / 6$
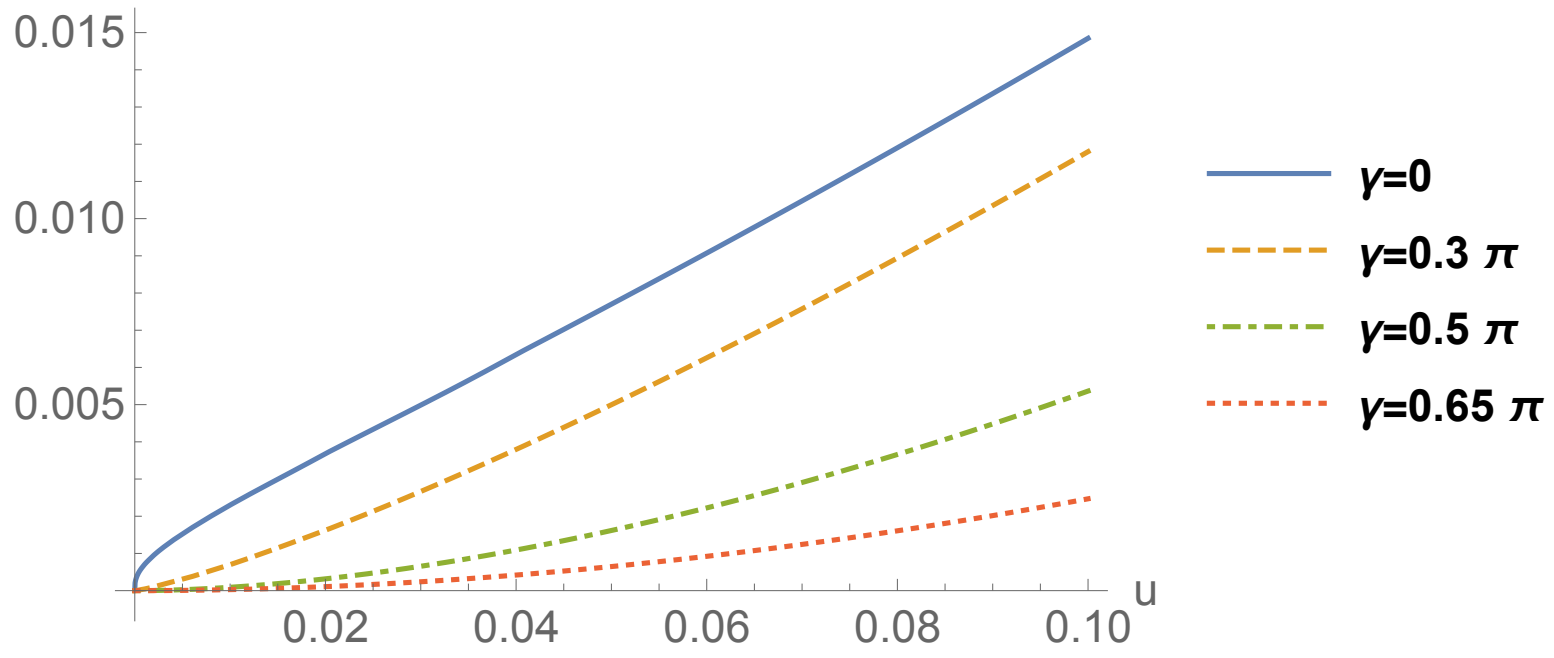

(b)

Figure 5: (a) Casimir scaling function $Y(u \mid \gamma)$, and (b) its deviation from the CFT value $-\frac{\pi}{6}$, plotted against the scaling parameter $u$ at different values of $\gamma$.

coefficient by fitting the numerical results for the sum

$$
Y(u \mid \gamma)+\frac{\pi}{6}-C_{1}(\gamma) u^{4 \gamma / \pi}+\frac{u^{2}}{4} \cot \left(\frac{\pi^{2}}{2 \gamma}\right)
$$

in the interval $0<u<0.05$.

Figure 7 displays the scaling function $Y(u \mid \gamma)+\pi / 6$ in the attractive regime at $\gamma=0.65 \pi$ in the region $0<u<1.5$. The black dots show the numerical data, the blue dashed line plots just one leading term in the small- $u$ asymptotic expansion (49), and the solid orange line displays the sum of two leading terms in this expansion. Note, that at $\gamma=0.65 \pi$ only two initial terms in the small- $u$ asymptotic expansion 49 describe 


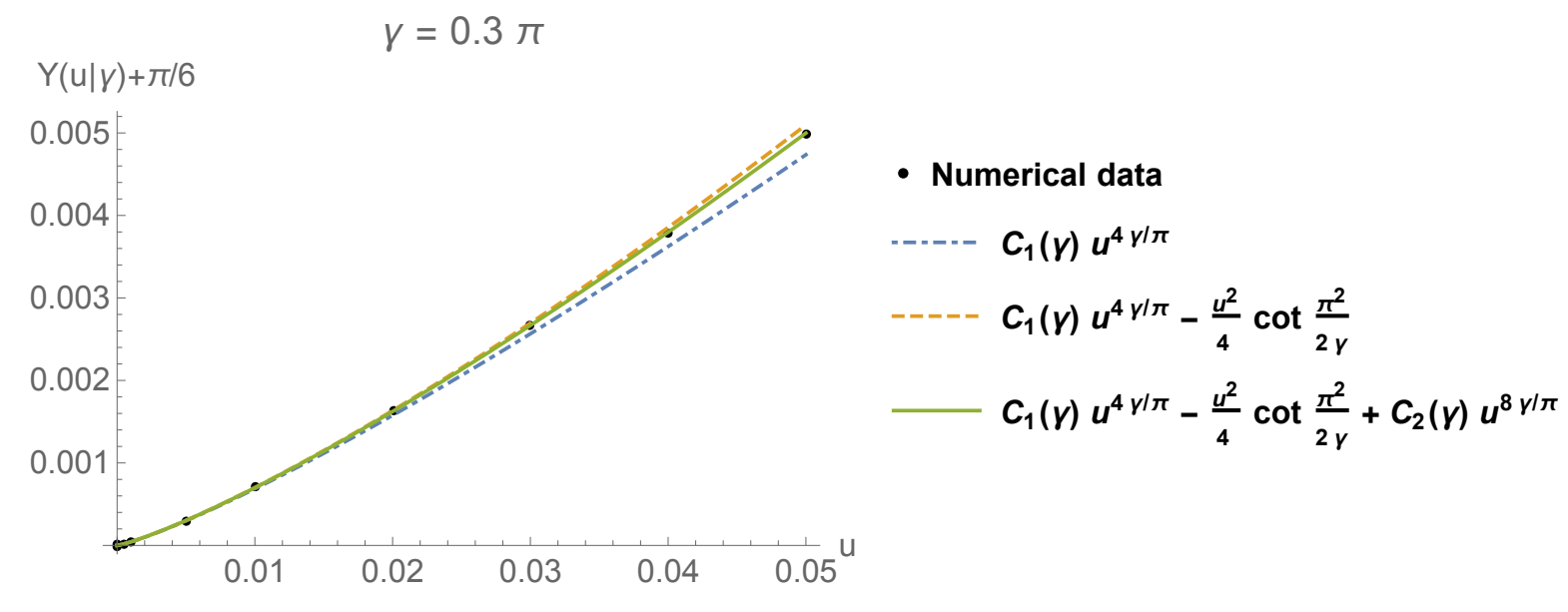

(a)

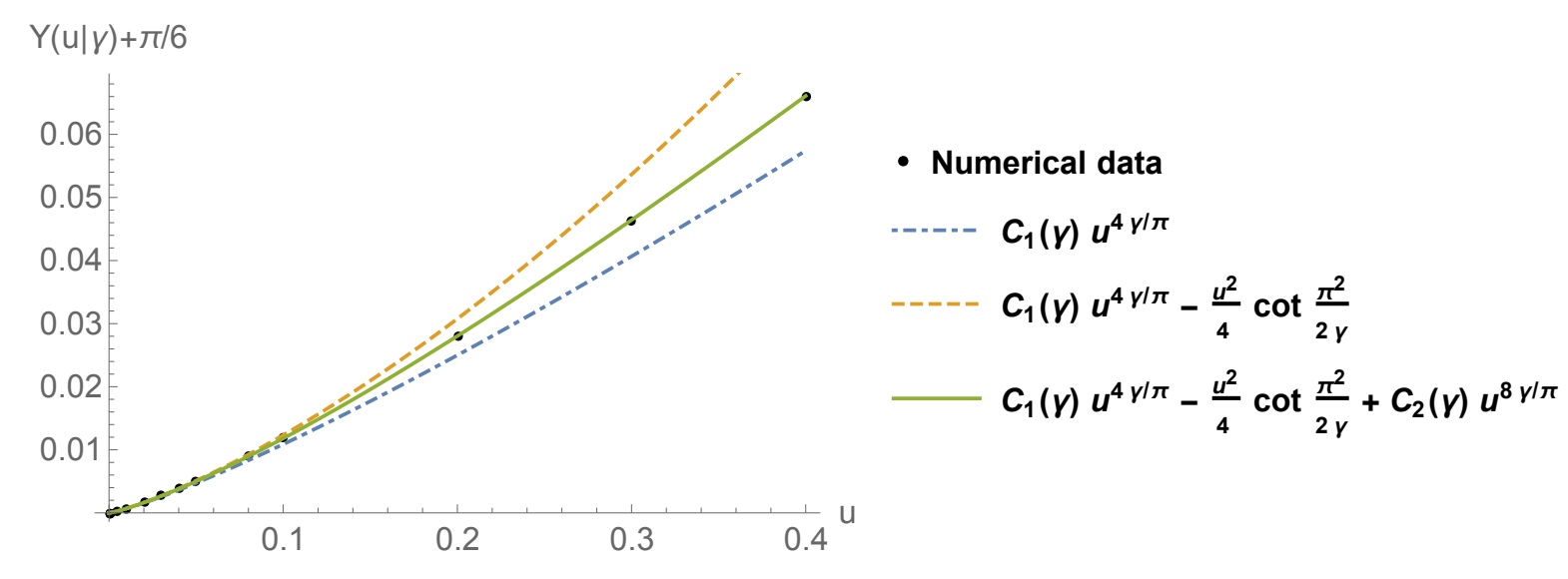

(b)

Figure 6: Casimir scaling function $Y(u \mid \gamma)+\frac{\pi}{6}$ in the repulsive regime at $\gamma=0.3 \pi$ plotted against the scaling parameter $u$ : (a) at $0<u<0.05$, (b) at $0<u<0.4$. Numerical data are shown by dots, The coefficient $C_{1}(\gamma)$ is given by (4), the coefficient $C_{2}(0.3 \pi)=-0.13$ was obtained by fitting the numerical data in the interval $0<u<0.05$.

remarkably accurate the numerical data for the Casimir scaling function in a very wide interval of the scaling parameter $u$.

\section{Acknowledgments}

A am thankful to Frank Göhmann and Andreas Klümper for helpful discussions.

\section{Appendix A. Derivation of (65) from the TBA equation}

In this Appendix, we perform the asymptotic analysis of the nonlinear TBA integral equations (26) at a small $u \rightarrow 0$, and obtain formula 65 for the Casimir scaling function 25) in the repulsive regime $\gamma \in(0, \pi / 2)$. The calculation is based on the 


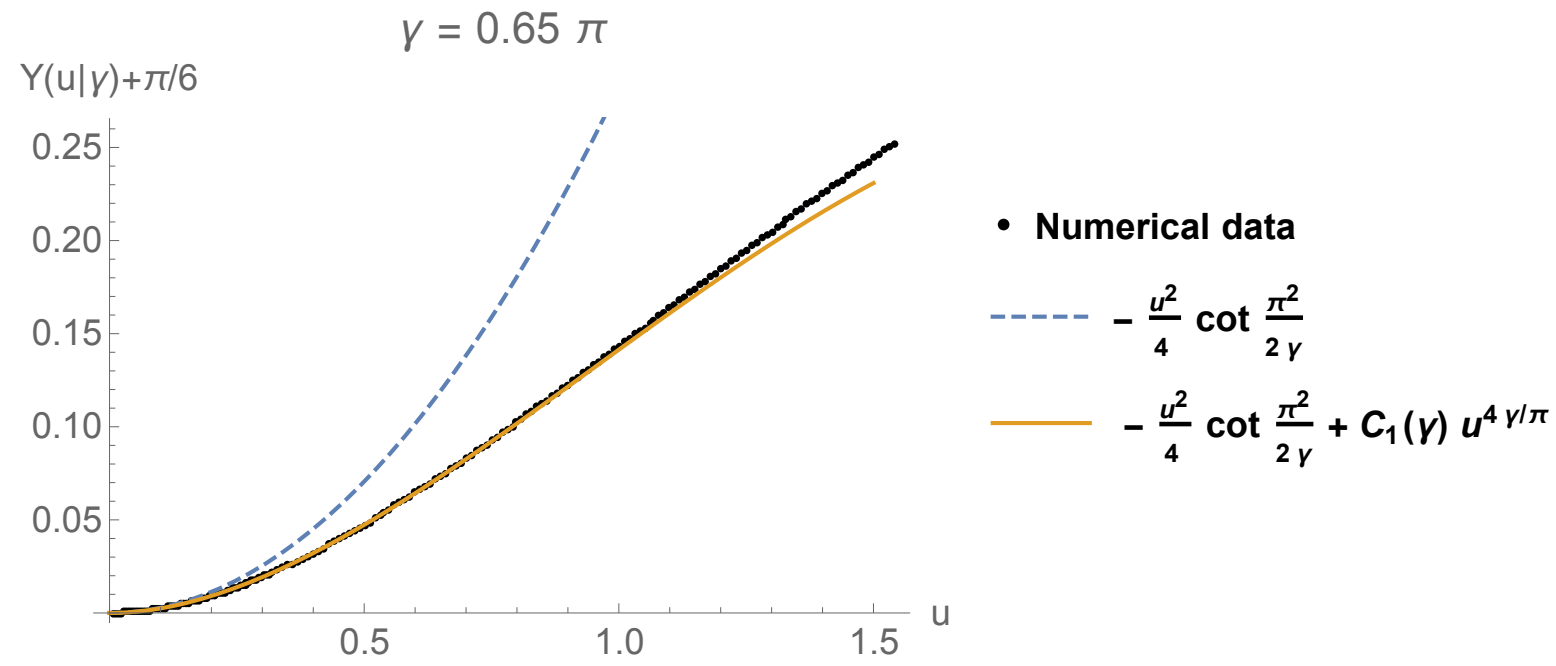

Figure 7: Casimir scaling function $Y(u \mid \gamma)+\frac{\pi}{6}$ in the attractive regime at $\gamma=0.65 \pi$ plotted against the scaling parameter $u$ at $0<u<1.5$. Numerical data are shown by dots. The dashed blue line displays the leading term in the small- $u$ asymptotics 49 of the scaling function. The solid orange line shows the plot of the two initial terms in the asymptotical formula (49) with the coefficient $C_{1}(\gamma)$ given by (4).

technique developed in [6].

Using the reflection symmetry (41) of the function $\varepsilon(\alpha \mid u, \gamma)$ and partial integration, we rewrite the integral formula $(25)$ for the scaling function in the form

$Y(u \mid \gamma)=-\frac{u}{\pi} \operatorname{Re} \int_{-\infty}^{\infty} d \alpha e^{\alpha} L(\alpha \mid u, \gamma)=\frac{u}{\pi} \operatorname{Re} \int_{-\infty}^{\infty} d \alpha e^{\alpha} \partial_{\alpha} L(\alpha \mid u, \gamma)$.

Then we divide the integral $\int_{-\infty}^{\infty} d \alpha$ on the right-hand side into two parts, $\int_{-\infty}^{\infty} d \alpha=$ $\int_{-\infty}^{0} d \alpha+\int_{0}^{\infty} d \alpha$. The first term is small $\sim u^{1+2 \gamma / \pi}$. Omitting this term, one obtains at $u \rightarrow 0$,

$$
Y(u)=\frac{2}{\pi} \operatorname{Re} \int_{0}^{\infty} d \alpha \frac{u e^{\alpha}}{2} \partial_{\alpha} L(\alpha \mid u, \gamma)+O\left(u^{1+2 \gamma / \pi}\right) .
$$

Let us now rewrite the TBA equation 27 as,

$$
\begin{aligned}
& \frac{u e^{\alpha}}{2}=\varepsilon(\alpha \mid u, \gamma)-\frac{u e^{-\alpha}}{2}+2 \int_{-\infty}^{\infty} d \alpha^{\prime} G\left(\alpha-\alpha^{\prime} \mid \gamma\right) \operatorname{Re} L\left(\alpha^{\prime} \mid u, \gamma\right) \\
& +\int_{-\infty}^{\infty} d \alpha^{\prime} g\left(\alpha-\alpha^{\prime}-i 0 \mid \gamma\right) L\left(\alpha^{\prime} \mid u, \gamma\right),
\end{aligned}
$$

substitute its right-hand side instead of the factor $u e^{\alpha} / 2$ into the integrand in A.2, and perform the term-wise integration of the resulting integral.

In the first term, the integration can be performed explicitly:

$$
\begin{aligned}
& J_{1}(u) \equiv-\frac{2}{\pi} \operatorname{Re} \int_{0}^{\infty} d \alpha \varepsilon(\alpha \mid u, \gamma) \frac{\partial_{\alpha} \varepsilon(\alpha \mid u, \gamma)}{1+\exp [\varepsilon(\alpha \mid u, \gamma)]}= \\
& -\frac{2}{\pi} \int_{\varepsilon(0 \mid u, \gamma)}^{\infty} d x \frac{x}{1+e^{x}}=-\frac{\pi}{6}+\frac{[\varepsilon(0 \mid u, \gamma)]^{2}}{2 \pi}+O\left([\varepsilon(0 \mid u, \gamma)]^{3}\right) .
\end{aligned}
$$


We use representation (42) for the pseudoenergy $\varepsilon(0 \mid u, \gamma)$, and drop in it the correction term $\eta(0 \mid u, \gamma)$, which does not contribute in A.5 to the leading order in $u$. Recalling, that $R(u)=\ln (2 / u)$ and using expansion (61), one obtains then:

$$
\begin{aligned}
& \varepsilon(0 \mid u, \gamma) \cong 2 \operatorname{Re} \varepsilon_{k}(-R(u) \mid u, \gamma)=\pi c_{1}(\gamma) \frac{\sin \gamma}{\gamma}\left(\frac{u}{2}\right)^{2 \gamma / \pi}+O\left(u^{4 \gamma / \pi}\right), \\
& J_{1}(u)=-\frac{\pi}{6}+\frac{\pi}{2}\left(\frac{c_{1}(\gamma) \sin \gamma}{\gamma}\right)^{2}\left(\frac{u}{2}\right)^{4 \gamma / \pi}+O\left(u^{6 \gamma / \pi}\right) .
\end{aligned}
$$

The second integral

$$
J_{2}(u) \equiv-\frac{2}{\pi} \operatorname{Re} \int_{0}^{\infty} d \alpha \frac{u e^{-\alpha}}{2} \partial_{\alpha} L(\alpha \mid u, \gamma)
$$

will be dropped, since it vanishes $\sim u^{1+2 \gamma / \pi}$ at $u \rightarrow 0$.

The sum of the two remaining double-integrals

$$
\begin{aligned}
& J_{3}(u) \equiv \frac{2}{\pi} \operatorname{Re}\left\{\int _ { 0 } ^ { \infty } d \alpha \partial _ { \alpha } L ( \alpha | u , \gamma ) \int _ { - \infty } ^ { \infty } d \alpha ^ { \prime } \left[2 G\left(\alpha-\alpha^{\prime} \mid \gamma\right) \operatorname{Re} L\left(\alpha^{\prime} \mid u, \gamma\right)\right.\right. \\
& \left.\left.+g\left(\alpha-\alpha^{\prime}-i 0 \mid \gamma\right) L\left(\alpha^{\prime} \mid u, \gamma\right)\right]\right\}
\end{aligned}
$$

can be represented as the sum of three terms:

$$
J_{3}(u)=A+B+C,
$$

where

$$
\begin{aligned}
& A=\frac{2}{\pi}[L(0 \mid u, \gamma)]^{2} \operatorname{Re} \int_{2 R(u)}^{\infty} d \alpha U(\alpha), \\
& B=-\frac{2}{\pi} L(0 \mid u, \gamma) \operatorname{Re} \int_{0}^{\infty} d \alpha U(\alpha+R(u)) \Psi(\alpha \mid u), \\
& C=\frac{4}{\pi} \iint_{0}^{\infty} d \alpha d \alpha^{\prime} G\left(\alpha+\alpha^{\prime} \mid \gamma\right) \operatorname{Re}\left[\partial_{\alpha} L(\alpha \mid u)\right] \operatorname{Re}\left[\Psi\left(\alpha^{\prime} \mid u\right)\right]+ \\
& \frac{2}{\pi} \operatorname{Re} \iint_{0}^{\infty} d \alpha d \alpha^{\prime} g\left(\alpha+\alpha^{\prime}-i 0 \mid \gamma\right) \partial_{\alpha} L(\alpha \mid u, \gamma) \Psi\left(\alpha^{\prime} \mid u\right),
\end{aligned}
$$

and

$$
\begin{aligned}
& U(\alpha)=2 G(\alpha \mid \gamma)+g(\alpha \mid \gamma) \\
& \Psi(\alpha \mid u)=L(\alpha \mid u, \gamma)-\Theta(R(u)-\alpha) L(0 \mid u, \gamma)
\end{aligned}
$$

Here $\Theta(x)$ denotes the unit-step function. Representation A.7 - A.10 for the integral $J_{3}(u)$ defined by $(\mathrm{A} .6)$ is exact. In the degenerate case $\gamma=0$, it was obtained in [6], see equations (A31), (A32) there.

We substitute the function $\varepsilon(\alpha \mid u, \gamma)$ in the form (42) into the integrals in (A.8), expand the result in appropriate fractional powers of $u$ using (61), and keep only the leading term $\sim u^{4 \gamma / \pi}$. It turns out, that:

(i) The correction term $\eta(\alpha \mid u, \gamma)$ in the right-hand side of 42 does not contribute to the above integrals to this order. 
(ii) The following estimates $A \sim u^{4 \gamma /(\pi-\gamma)}, B \sim u^{2 \gamma / \pi+2 \gamma /(\pi-\gamma)}, C \sim u^{4 \gamma / \pi}$ hold for these integrals. Therefore, in the considered regime $0<\gamma<\pi / 2$, the integrals $A$ and $B$ can be dropped in A.7 to the leading order in $u$.

(iii) The main contribution into the double-integrals in $\mathrm{A} .10$ comes from finite $\alpha, \alpha^{\prime} \sim$ 1. At finite $\alpha, \alpha^{\prime} \ll R(u)$, the following substitutions can be safely made in the right-hand side of (A.10):

$$
\begin{aligned}
& \partial_{\alpha} L(\alpha \mid u, \gamma)=-\frac{\partial_{\alpha} \varepsilon(\alpha \mid u, \gamma)}{2}+O\left(u^{4 \gamma / \pi}\right), \quad \Psi(\alpha \mid u)=-\frac{\varepsilon(\alpha \mid u, \gamma)}{2}+O\left(u^{4 \gamma / \pi}\right), \\
& \varepsilon(\alpha \mid u, \gamma)=\frac{\pi i}{2 \gamma} c_{1}(\gamma)\left(\frac{u}{2}\right)^{2 \gamma / \pi}\left[\exp \left(\frac{2 \gamma \alpha}{\pi}+i \gamma\right)-\exp \left(-\frac{2 \gamma \alpha}{\pi}-i \gamma\right)\right]+O\left(u^{4 \gamma / \pi}\right) .
\end{aligned}
$$

The resulting double-integrals in A.10 can be easily calculated using equalities (4). This yields:

$$
C=\frac{\pi c_{1}(\gamma)^{2}}{2 \gamma^{2}}\left(\frac{\pi}{2 \sin 2 \gamma}-\sin ^{2} \gamma\right)
$$

Combining (A.13) with A.5 and A.7), we arrive at the final result (65).

\section{Appendix B. Perturbative CFT calculation of $Y(u)$}

The free energy $f(\mu, L)$ per unit area of the sine-Gordon model in the infinite strip of width $L$ is defined as the limit

$$
f(\mu, \gamma, L)=-\lim _{L^{\prime} \rightarrow \infty} \frac{\left.\ln Z\left(\mu, \gamma, L, L^{\prime}\right)\right]}{L L^{\prime}},
$$

where the partition function $Z\left(\mu, L, L^{\prime}\right)$ on the torus $\mathcal{T}$ is determined by the continual integral (17). Expansion of this integral to the second order in $\mu$ yields

$$
\begin{aligned}
& -f(\mu, \gamma, L)=-f(0, \gamma, L)+2 \mu\langle\cos [\beta \varphi(0)]\rangle+ \\
& 2 \mu^{2} \int_{-\infty}^{\infty} d x^{0} \int_{0}^{L} d x^{1}\langle\cos [\beta \varphi(x)] \cos [\beta \varphi(0)]\rangle \Theta(|x|-a)+O\left(\mu^{4}\right),
\end{aligned}
$$

where the correlation functions are connected and calculated at $\mu=0$. The cut-off at the lattice constant $a$ serves to regularise the integral at short distances. The linear in $\mu$ term in the right-hand side vanishes, since $\langle\cos [\beta \varphi(0)]\rangle=0$. The second-order term $\delta_{2} f(\mu, L)$ can be written as

$\delta_{2} f(\mu, \gamma, L)=-\mu^{2} \int_{-\infty}^{\infty} d x^{0} \int_{0}^{L} d x^{1}\langle\exp [i \beta \varphi(x)] \exp [-i \beta \varphi(0)]\rangle \Theta(|x|-a)$.

For the strip geometry, the correlation function of the exponential operators in the integrand can be determined [27] by use of the conformal invariance at $\mu=0$,

$$
\left\langle e^{i \beta \varphi(x)} e^{-i \beta \varphi(0)}\right\rangle=\left(\frac{2 \pi}{L}\right)^{2 \mathrm{x}}\left\{2\left[\cosh \left(2 \pi x^{0} / L\right)-\cos \left(2 \pi x^{1} / L\right)\right]\right\}^{-\mathrm{x}},
$$

with the scaling dimension $\mathrm{x}$ given by (7). After substitution of this expression into (B.3) and rescaling the integration variables, one finds,

$$
\delta_{2} f(\mu, \gamma, L)=-\mu^{2}\left(\frac{2 \pi}{L}\right)^{2 \mathrm{x}-2} I_{2}(\mathrm{x}, \varepsilon),
$$


where

$$
I_{2}(\mathrm{x}, \varepsilon)=\int_{-\infty}^{\infty} d t \int_{0}^{2 \pi} d \theta \frac{1}{[2(\cosh t-\cos \theta)]^{\mathrm{x}}} \Theta\left(\sqrt{t^{2}+\theta^{2}}-\varepsilon\right),
$$

and $\varepsilon=2 \pi a / L$.

At $0<\mathrm{x}<1$, the parameter $\beta^{2}$ lies in the interval $(0,1 / 2)$, and the attractive regime of the sine-Gordon model is realised. The integral $I_{2}(\mathrm{x}, 0)$ converges in this case. It's explicit expression was obtained by Hentschke et al. [28,

$$
I_{2}(\mathrm{x}, 0)=\pi \frac{\Gamma^{2}\left(\frac{1}{2} \mathrm{x}\right) \Gamma(1-\mathrm{x})}{\Gamma^{2}\left(1-\frac{1}{2} \mathrm{x}\right) \Gamma(\mathrm{x})}
$$

At a small $\varepsilon>0$, the leading behaviour of the integral (B.6) reads [7],

$$
I_{2}(\mathrm{x}, \varepsilon)=I_{2}(\mathrm{x}, 0)-\frac{\pi \varepsilon^{2-2 \mathrm{x}}}{1-\mathrm{x}}+O\left(\varepsilon^{6-2 \mathrm{x}}\right) .
$$

Equations (B.5), (B.7), (B.8) can be used in both $0<\mathrm{x}<1$ and $1<\mathrm{x}<2$ cases, which correspond to the attractive and repulsive regimes of the sine-Gordon model, respectively. Substitution of (B.8) into (B.5) yields,

$$
\delta_{2} f(\mu, \gamma, L)=-\mu^{2}\left(\frac{2 \pi}{L}\right)^{2 \mathrm{x}-2} I_{2}(\mathrm{x}, 0)+\frac{\pi \mu^{2}}{1-\mathrm{x}} a^{2-2 \mathrm{x}} .
$$

In the repulsive regime $1<\mathrm{x}<2$, the $L$-independent term in the right-hand side diverges at $a \rightarrow 0$ and contributes to the non-universal part of the bulk free energy.

Let us now use equation $(10)$ to express the coupling constant $\mu$ in equation (B.9) in terms of the soliton mass $m$,

$\delta_{2} f[\mu(m), \gamma, L]=-\kappa(\xi)^{2} m^{4 /(\xi+1)}\left[\left(\frac{2 \pi}{L}\right)^{2 \mathrm{x}-2} I_{2}(\mathrm{x}, 0)-\frac{\pi}{1-\mathrm{x}} a^{2-2 \mathrm{x}}\right]$.

After replacement of parameters $\xi$ and $\mathrm{x}$ in this equation by their expressions in terms of the parameter $\gamma$,

$$
\xi=\frac{\pi-\gamma}{\gamma}, \quad \mathrm{x}=\frac{2(\pi-\gamma)}{\pi}
$$

one obtains

$$
\delta_{2} f[\mu(m), \gamma, L]=\frac{C_{1}(\gamma)}{L^{2}} u^{4 \gamma / \pi}-\frac{\pi^{2}}{(\pi-2 \gamma) a^{2}}(m a)^{4 \gamma / \pi},
$$

where $u=m L$ is the scaling parameter, and $C_{1}(\gamma)$ is given by (4).

Let us recall now that due to equation (18) the ground-state energy $E(\mu, \gamma, L)$ of the sine-Gordon model Hamiltonian in the circle of length $L$ is proportional to the free energy $f(\mu, \gamma, L)$ per unit area,

$$
E(\mu, \gamma, L)=L f(\mu, \gamma, L)
$$

Combining this with equality (19) allows one to relate the Casimir scaling function $Y(u \mid \gamma)$ with $f(\mu, \gamma, L)$ and the bulk energy density $\mathcal{E}_{b}(\mu, \gamma)$.

$$
Y(u \mid \gamma)=L^{2}\left[f(\mu, \gamma, L)-\mathcal{E}_{b}(\mu, \gamma)\right]
$$


The energy density of the slab $f(\mu, \gamma, L)$, as well as the bulk energy density in the sine-Gordon model

$$
\mathcal{E}_{b}(\mu, \gamma)=\lim _{L \rightarrow \infty} f(\mu, \gamma, L)
$$

contain the ultraviolet-diverging parts, and the (independent of the lattice spacing $a$ ) scaling parts. The explicit expression of the scaling part $\mathcal{E}_{b}^{(s c)}[\mu(m), \gamma]$ of the bulk energy density is well known [29, 15],

$$
\mathcal{E}_{b}^{(s c)}[\mu(m), \gamma]= \begin{cases}\frac{m^{2}}{4} \cot \left(\frac{\pi^{2}}{2 \gamma}\right), & \text { if } \gamma \neq \frac{\pi}{2 n}, \\ \frac{m^{2}}{2 \pi n} \ln m, & \text { if } \gamma=\frac{\pi}{2 n},\end{cases}
$$

where $m$ is the soliton mass 100 , and $n=1,2, \ldots$. Since the universal Casimir scaling function $Y(u \mid \gamma)$ in the left-hand side of (B.13) is ultraviolet-convergent, the same is true for the right-hand side of equation (B.13). This implies, in particular, that the second ( $L$-independent, ultraviolet-diverging) term in the right-hand side of (B.11) must cancel with the same contribution from the bulk energy density $\mathcal{E}_{b}(\mu, \gamma)$ in brackets in the right-hand-side of (B.13). So, we can replace the functions $f(\mu, \gamma, L)$ and $\mathcal{E}_{b}(\mu, \gamma)$ in the right-hand side of $(\overline{\mathrm{B} .13})$ by their scaling counterparts

$$
\begin{aligned}
& f^{(s c)}(u, \gamma, L)=\frac{1}{L^{2}}\left[-\frac{\pi}{6}+C_{1}(\gamma) u^{4 \gamma / \pi}+O\left(u^{8 \gamma / \pi}\right)\right], \text { if } \gamma \neq \frac{\pi}{2}, \\
& \mathcal{E}_{b}^{(s c)}(u, \gamma, L)= \begin{cases}\frac{u^{2}}{4 L^{2}} \cot \left(\frac{\pi^{2}}{2 \gamma}\right), & \text { if } \gamma \neq \frac{\pi}{2 n}, \\
\frac{u^{2}}{2 \pi n L^{2}} \ln u, & \text { if } \gamma=\frac{\pi}{2 n},\end{cases}
\end{aligned}
$$

with $n=1,2, \ldots$. This leads to the results (49), (51), that hold at $\gamma \neq \pi / 2$.

The Casimir scaling function degenerates in the free-fermionic case $\gamma=\pi / 2$ to the form,

$$
Y(u \mid \pi / 2)=-\frac{u}{\pi} \int_{-\infty}^{\infty} d \alpha \cosh \alpha \ln \left[1+e^{-u \cosh \alpha}\right] .
$$

Its behaviour at small $u \rightarrow 0$ is described by equation (50).

\section{Appendix C. Calculation of the coefficient $c_{1}(\gamma)$ in the attractive regime} $\pi / 2<\gamma<\pi$

The DDV integral equation (3.14) studied in [4] relates to the massless case $\mu \rightarrow 0$ of the sine-Gordon model (5) perturbed by the uniform gauge "magnetic field" $p$, which is coupled to the soliton charge. At $p=0$, this equation reduces to the form:

$$
i \ln a(\theta)=2 M \cos \frac{\pi \xi}{2} e^{\theta}-2 \int_{-\infty}^{\infty} d \theta^{\prime} G\left(\theta-\theta^{\prime}\right) \operatorname{Im} \ln \left[1+a\left(\theta^{\prime}-i 0\right)\right]
$$

where the integral kernel $G(\theta)$ is given by $(22)$, the constant $M$ reads

$$
M=\frac{\Gamma\left(\frac{\xi}{2}\right) \Gamma\left(\frac{1}{2}-\frac{\xi}{2}\right)}{\sqrt{\pi}}\left(\Gamma\left(1-\beta^{2}\right)\right)^{1+\xi},
$$


and parameters $\xi$ and $\beta$ are related with $\gamma$ according to (6). Upon the shift of the rapidity variable

$$
\alpha=\theta+\ln \left(2 M \cos \frac{\pi \xi}{2}\right)
$$

and the substitution

$$
a(\theta)=\frac{1}{\mathfrak{f}_{k}(\alpha)},
$$

equation C.1 transforms to the form (63).

It was shown in [4], that the function $a(\theta)$ solving equation (C.1) admits in the attractive case $0<\xi<1$ the following representation:

$$
a(\theta)=\frac{A(\lambda q)}{A\left(\lambda q^{-1}\right)}
$$

where $\lambda=\exp \left(\frac{\theta}{1+\xi}\right), q=\exp \left(\frac{i \pi \xi}{1+\xi}\right)$, and $A(\lambda)$ is the vacuum eigenvalue of the operator $\mathrm{Q}_{+}(\lambda)$, which is the CFT analogue of the $Q$-matrix introduced by Baxter [30, 31, 32, 33]. The function $A(\lambda)$ has remarkable analytical properties. It was shown in [4], in particular, that $A(\lambda)$ is an entire function in the attractive regime $0<\xi<1$, that can be represented in this case by a convergent product

$$
A(\lambda)=\prod_{k=0}^{\infty}\left(1-\frac{\lambda^{2}}{\lambda_{k}^{2}}\right),
$$

and all zeroes $\lambda_{k}^{2}$ of $A(\lambda)$ are positive. Accordingly, the function $\phi(\theta)=i \ln a(\theta)$ admits at $\theta \rightarrow-\infty$ the converging Taylor expansion in $\lambda$ :

$$
\phi(\theta)=\sum_{n=1}^{\infty} b_{n} \lambda^{2 n}
$$

with real coefficients $b_{n}$, and

$$
b_{1}=2 \sin \left(\frac{2 \pi \xi}{1+\xi}\right) \sum_{k=1}^{\infty} \lambda_{k}^{-2} .
$$

The explicit formula for the infinite sum in the right-hand side can be gained from equations (3.21), (2.34) and (2.35) in [4]:

$$
\sum_{k=1}^{\infty} \lambda_{k}^{-2}=\Gamma\left(1-2 \beta^{2}\right)\left[\Gamma\left(\beta^{2}\right)\right]^{2} .
$$

The resulting expression for the coefficient $b_{1}$ reads:

$$
b_{1}=2 \sin \left(\frac{2 \pi \xi}{1+\xi}\right) \Gamma\left(1-2 \beta^{2}\right)\left[\Gamma\left(\beta^{2}\right)\right]^{2} .
$$

On the other hand, the functions $\phi(\theta)$ and $\phi_{k}(\alpha)$ coincide after the shift (C.3) of the rapidity argument: $\phi(\theta)=\phi_{k}(\alpha)$. Therefore, expansion 64 represents the analytical continuation (in the parameter $\xi$ ) of the Taylor expansion (C.7) into the 
interval $\xi \in(1,+\infty)$, which corresponds to the repulsive regime of the sine-Gordon model. This leads to the simple relation between the coefficients of these two expansions:

$$
c_{n}(\gamma)=\frac{2 n \gamma}{\pi} b_{n}\left[2 M \cos \frac{\pi(\pi-\gamma)}{2 \gamma}\right]^{-2 n \gamma / \pi} .
$$

Taking (C.10) into account, we arrive at the following representation for the first coefficient

$c_{1}(\gamma)=-\frac{4 \gamma}{\pi} \sin (2 \gamma) \Gamma\left(\frac{2 \gamma}{\pi}-1\right)\left[\Gamma\left(1-\frac{\gamma}{\pi}\right)\right]^{2}\left[2 M \cos \frac{\pi(\pi-\gamma)}{2 \gamma}\right]^{-2 \gamma / \pi}$,

which is equivalent to (66).

\section{References}

[1] G. Mussardo. Statistical Field Theory: An Introduction to Exactly Solved Models in Statistical Physics. Oxford University Press, Oxford, 2010.

[2] Al. B. Zamolodchikov. Thermodynamic Bethe ansatz in relativistic models: Scaling 3-state Potts and Lee-Yang models. Nuclear Physics B, 342(3):695 - 720, 1990.

[3] V. V. Bazhanov, S. L. Lukyanov, and A. B. Zamolodchikov. Integrable structure of conformal field theory, quantum KdV theory and Thermodynamic Bethe Ansatz. Comm. Math. Phys., 177:381-398, 1996.

[4] V. V. Bazhanov, S. L. Lukyanov, and A. B. Zamolodchikov. Integrable structure of Conformal Field Theory II. Q-operator and DDV equation. Comm. Math. Phys., 190:247-278, 1997.

[5] Al. B. Zamolodchikov. Resonance factorized scattering and roaming trajectories. Journal of Physics A: Mathematical and General, 39(41):12847-12861, 2006.

[6] S. B. Rutkevich. Scaling in the massive antiferromagnetic $X X Z$ spin-1/2 chain near the isotropic point. Phys. Rev. E, 101:032115, 2020.

[7] J. L. Cardy. Logarithmic corrections to finite-size scaling in strips. Journal of Physics A: Mathematical and General, 19(17):L1093-L1098, 1986.

[8] I. Affleck, D. Gepner, H. J. Schulz, and T. Ziman. Critical behaviour of spin-s Heisenberg antiferromagnetic chains: analytic and numerical results. Journal of Physics A: Mathematical and General, 22(5):511-529, 1989.

[9] S. Lukyanov. Low energy effective Hamiltonian for the XXZ spin chain. Nuclear Physics B, 522(3):533 - 549, 1998.

[10] G. Feverati, F. Ravanini, and G. Takács. Truncated conformal space at $c=1$, nonlinear integral equation and quantization rules for multi-soliton states. Physics Letters B, 430(3):264 - 273, 1998.

[11] G. Feverati, F. Ravanini, and G. Takács. Scaling functions in the odd charge sector of sineGordon/massive Thirring theory. Physics Letters B, 444(3):442 - 450, 1998.

[12] A. Klümper and M. T. Batchelor. An analytic treatment of finite-size corrections in the spin-1 antiferromagnetic XXZ chain. Journal of Physics A: Mathematical and General, 23(5):L189L195, 1990.

[13] A. Klümper, M. T. Batchelor, and P. A. Pearce. Central charges of the 6- and 19-vertex models with twisted boundary conditions. Journal of Physics A: Mathematical and General, 24(13):3111-3133, 1991.

[14] C. Destri and H. J. de Vega. New thermodynamic Bethe ansatz equations without strings. Phys. Rev. Lett., 69:2313-2317, 1992.

[15] C. Destri and H. J. de Vega. Unified approach to Thermodynamic Bethe Ansatz and finite size corrections for lattice models and field theories. Nuclear Physics B, 438(3):413 - 454, 1995. 
[16] A. Klümper. Free energy and correlation lengths of quantum chains related to restricted solid-onsolid lattice models. Annalen der Physik, 504(7):540 - 553, 1992.

[17] D. Fioravanti, A. Mariottini, E. Quattrini, and F. Ravanini. Excited state Destri-de Vega equation for sine-Gordon and restricted sine-Gordon models. Physics Letters B, 390(1):243 - 251, 1997.

[18] C. Destri and H. J. de Vega. Non-linear integral equation and excited-states scaling functions in the sine-Gordon model. Nuclear Physics B, 504(3):621 - 664, 1997.

[19] G. Feverati, F. Ravanini, and G. Takács. Non-linear integral equation and finite volume spectrum of sine-Gordon theory. Nuclear Physics B, 540(3):543 - 586, 1999.

[20] P. Zinn-Justin. Nonlinear integral equations for complex affine Toda models associated with simply laced Lie algebras. Journal of Physics A: Mathematical and General, 31(31):6747-6770, 1998.

[21] G. Feverati, F. Ravanini, and G. Takács. Non-linear integral equation and finite volume spectrum of minimal models perturbed by $\Phi_{(1,3)}$. Nuclear Physics B, 570(3):615 - 643, 2000.

[22] Á. Hegedüs. Finite volume expectation values in the sine-Gordon model. J. High Energ. Phys., 2020:122, 2020.

[23] Al. B. Zamolodchikov. Mass scale in the sine-Gordon model and its reductions. Int. Journ. of Mod. Phys. A, 10(8):1125 - 1150, 1995.

[24] A. B. Zamolodchikov. Exact two-particle $S$-matrix of quantum sine-Gordon solitons. Commun. Math. Phys., 55(2):183 - 186, 1977.

[25] F. A. Smirnov. Form-factors in completely integrable models of quantum field theory, (Advanced Series in Mathematical Physics, Vol. 14). World Scientific, Singapore, 1992.

[26] D. Fioravanti and M. Rossi. From finite geometry exact quantities to (elliptic) scattering amplitudes for spin chains: the 1/2-XYZ. Journal of High Energy Physics, 2005(08):010-010, 2005.

[27] J. L. Cardy. Conformal invariance and universality in finite-size scaling. Journal of Physics A: Mathematical and General, 17(7):L385-L387, 1984.

[28] R. Hentschke, P. Kleban, and G. Akinci. Structure function, susceptibility and correlation lengths at critical points for infinite strips with periodic boundary conditions. Journal of Physics A: Mathematical and General, 19(16):3353-3359, 1986.

[29] R. J. Baxter. One-dimensional anisotropic Heisenberg chain. Annals of Physics, 70(2):323 - 337, 1972.

[30] R. J. Baxter. Eight-vertex model in lattice statistics and one-dimensional anisotropic Heisenberg chain. I. Some fundamental eigenvectors. Annals of Physics, 76(1):1 - 24, 1973.

[31] R. J. Baxter. Eight-vertex model in lattice statistics and one-dimensional anisotropic Heisenberg chain. II. Equivalence to a generalized ice-type lattice model. Annals of Physics, 76(1):25 - 47, 1973.

[32] R. J. Baxter. Eight-vertex model in lattice statistics and one-dimensional anisotropic Heisenberg chain. III. Eigenvectors of the transfer matrix and hamiltonian. Annals of Physics, 76(1):48 $71,1973$.

[33] R. J. Baxter. Exactly solved models in statistical mechanics. Academic Press, London, 1982. 